\title{
STUDY OF THE FORCE COEFFICIENTS ON PLATES USING AN OPEN SOURCE NUMERICAL WAVE TANK
}

\author{
Krishakumar Rajagopalan*, Gérard Nihous \\ Dept. of Ocean and Resources Engineering, University of Hawaii, Honolulu, HI 96822, U.S.A.
}

\begin{abstract}
An open source numerical wave tank based on the OpenFOAM@ software was setup and tested to solve the Navier-Stokes equations. This work focused on submerged plates because of their simple geometry, their importance as a design component (e.g., heave plates), and the broad availability of published experimental results. Two-dimensional plates in an oscillatory flow, as well as three-dimensional circular disks and square plates undergoing forced oscillations were simulated in conditions where the nonlinearity of hydrodynamic viscous drag and inertia forces can be probed easily. The effect of plate thickness as well as the ability of the numerical simulations to predict wave radiation damping at shallow plate submergence were also considered. Overall, force coefficient predictions were very satisfactory when compared to experimental data, and generally exhibited less scatter. The influence of both Keulegan-Carpenter number and frequency parameter on the hydrodynamic force could be assessed as well from these numerical simulations as from past laboratory experiments.
\end{abstract}

Keywords: numerical wave tank; drag coefficient; inertia coefficient.

* Corresponding author. Tel. +1 8083973444; E-mail address: krishnak@ hawaii.edu 


\section{Introduction}

The solution of the Navier-Stokes equations, subject to specific boundary conditions, has been a formidable task for generations of researchers in the field of fluid mechanics. In truth, simplified theories have often been, and remain, very fruitful, such as those based on velocity potentials, when assumptions of inviscid fluids and irrotational flows hold, or on boundary layer representations. Tackling more complex problems where viscosity and turbulence play a significant role, however, generally does not afford shortcuts, and extensive experimentation may be required. For practical and economic reasons, results at laboratory scale may have to be interpreted invoking similarity scaling laws.

The recent advent of unprecedented computing capabilities has allowed new opportunities in modeling fluid flow, and the field of computational fluid dynamics (CFD) is booming. Software suites have been developed to a great level of sophistication, and CFD codes have become broadly available, either under commercial licensing or as open source resources. Thus, the prospect of leveraging numerical wave tanks (NWTs) to a much greater extent in composite modeling, which calls for a balanced use of physical and numerical models (Gerritsen and Sutherland 2011), has become a reality, even though NWT development is a challenging task in itself, and that the same standards of validation and verification must be applied as with any theoretical and experimental work. This is often accomplished by benchmarking, i.e. by solving the same specific problems with different tools before comparing the results to known solutions. More loosely, an NWT can be tested against any combination of available theoretical, numerical or experimental results. The purpose of the present work is to present preliminary tests of an NWT based on the open source (free) software OpenFOAM®. The initial motivation 
behind this effort is to be able to use the NWT effectively in modeling and developing wave energy converters (WECs). A recent review of numerical methods considered in the analysis of WECs ( $\mathrm{Li}$ and $\mathrm{Yu}, 2012$ ) shows that there is a distinct hierarchy of models for this purpose, from linear potential flow theory to the numerical solution of the NavierStokes equations. There is a clear tradeoff between computing time and accuracy: if the assumptions of linear potential flow theory hold well, it would be unwise to use an NWT, especially in a design phase that may involve a very large number of cases (device geometries, wave conditions, power absorption mechanisms etc). If the flow is such that basic assumptions of linear potential flow theory were violated in ways that significantly affect WEC evaluation, the ultimate use of an NWT would be justified, as in the study of a two-body WEC point absorber by $\mathrm{Yu}$ and $\mathrm{Li}$ (2013), who used the commercial CFD software STAR-CCM+®.

While overall WEC modeling in an NWT remains difficult and time consuming, though not necessarily more so than experimental work, a widely used and very efficient intermediate modeling level is available. As first proposed by Cummins (1962), linear wave hydrodynamic results from frequency-domain potential theory can be used in a time domain model; this is accomplished with impulse response functions in convolution integrals, a methodology summarized for example in Sheng and Lewis (2012). Some nonlinear fluid effects can then be added, like viscous drag in a simplified quadratic term, but also external loads that are not oscillatory (harmonic); the latter commonly arise in the power take off (PTO) mechanisms of WECs. A thorough numerical benchmarking study of WECs based on this efficient time-domain approach was recently published (Babarit et al., 2012). Yet, the addition of nonlinear fluid flow features in such models 
may not be straightforward. Only if simple geometries like circular cylinders are considered and motions follow typical patterns (e.g., oscillations) might the technical literature provide sufficient information on inertia and viscous forces (e.g., Sarpkaya and Isaacson, 1981; Molin, 2002). As Babarit et al. (2012) wrote, "the estimation of the drag coefficient was identified as the main error source for almost all of the [WEC] devices". Li and Yu (2012) admitted that for practical WEC designs, "hydrodynamic forces may have to be evaluated by conducting additional wave tank tests or prescribed motion CFD simulations". Thus, a practical role for NWTs in WEC evaluation may very well be a supporting one, at least in the short term, in which salient system nonlinearities would be analyzed separately. This suggests that the modeling of standard shapes with imposed motions or in well defined flows not only represents a useful preliminary step in validating NWTs, but could also provide valuable information to simpler WEC models. Accordingly, the present work focuses on the hydrodynamic behavior of simple plates. The case of fixed two-dimensional submerged plates in oscillating flows is considered next in Section 2, while three-dimensional submerged plates undergoing prescribed oscillations are analyzed in Section 3. It is further pointed out that all the plates considered in this study are rigid and that the parameters of the numerical experiments in all cases are carefully chosen such that wave breaking does not occur. Concluding remarks are summarized in Section 4.

\section{Oscillating flow over fixed submerged two-dimensional plates}

2.1 Measurements (Keulegan and Carpenter, 1958) 
The hydrodynamic forces acting on a body in an oscillating, unbounded, real fluid can be divided into a component in phase with fluid acceleration and a component in phase with fluid velocity, as noted by Keulegan and Carpenter (1958). A non-dimensional inertia coefficient $C_{m}$ can be defined, which is associated with the acceleration of the flow, as well as a non-dimensional drag coefficient $C_{d}$, which is associated with the velocity of the flow. Keulegan and Carpenter (1958) found that $C_{m}$ and $C_{d}$ both depend on a particular non-dimensional group, the Keulegan-Carpenter number $K C=U_{m} T / D$, where $U_{m}$ represents the amplitude of the oscillating velocity in a direction perpendicular to the plate, $T$ the time period of the oscillation and $D$ the dimension of the plate. In order to create an oscillatory flow, Keulegan and Carpenter (1958) generated stationary waves in a wave tank and placed the flat plates vertically in the nodal region. These waves were well described with a second-order wave theory. The wave tank was $2.42 \mathrm{~m}$ long, $0.7 \mathrm{~m}$ deep and the plates were located $0.25 \mathrm{~m}$ below the still water level. The wave period was held constant at $2.075 \mathrm{~s}$ throughout the tests. By changing the height of the plates and the amplitude of the waves, which in turn changed $U_{m}$, total force measurements per unit width of the plates were obtained for a range of $K C$ from 2 to 125 . Fourier analysis was then applied to estimate $C_{m}$ and $C_{d}$ as given in Equations 30 and 31 of Keulegan and Carpenter (1958). Since the focus of their study was two-dimensional flow, the width of both tank and plates was not of particular interest.

\subsection{Numerical experiments with OpenFOAM}

The OpenFOAM CFD solver with the wave generation tool box (waves2Foam) described in Jacobsen (2011a) and summarized in Section 2 of Jacobsen et al. (2011b), was selected to numerically reproduce the experimental work of Keulegan and Carpenter (1958). In 
waves 2 Foam, wave making is achieved by specifying pressures and velocities on the wave generating boundary. This however requires a relaxation region next to this boundary to ensure that internally reflected waves do not contaminate wave generation and create discontinuities at the free surface. Such a strategy contrasts with numerical wave tanks where waves are generated by a moving boundary, as in a physical wave tank (Higuera et. al 2015). Although the latter approach represents wave makers more explicitly, the implementation of a moving boundary is not trivial. Moreover the methodology adopted by Jacobsen (2011a) effectively renders the details and complications of a physical wave generation systems less relevant.

In this work second-order stream-function waves of period $2.075 \mathrm{~s}$ were selected as input to the numerical wave tank. The numerical domain was $0.7 \mathrm{~m}$ deep and $19.68 \mathrm{~m}$ (four wavelengths) long with $9.84 \mathrm{~m}$ (two wavelengths) assigned to the wave relaxation region adjacent to the wave generation boundary. At the other end of the tank, a reflecting wall allowed the development of a stationary wave field in the tank. It should be noted that in this respect, standing wave generation is simpler than progressive wave generation since the reflecting wall replaces what would be an absorption boundary. In all the numerical experiments, the vertical plate was located with its center $0.25 \mathrm{~m}$ below the free surface at the nodal region of the stationary wave. As the thickness of the plate is not given in Keulegan and Carpenter (1958), it was inferred from Figure 30 of their paper to be $4 \mathrm{~mm}$. An important quantity used to normalize the inertia and drag coefficients is the horizontal velocity amplitude $U_{m}$. In Keulegan and Carpenter (1958), $U_{m}$ was estimated analytically. In the OpenFOAM experiments, it was measured one node away from the plate, after 
verifying that the same value would be obtained at the plate location with the plate removed.

As the Reynolds number, $R e=U_{m} D / v$, where $v$ is the kinematic viscosity of water, is small in these experiments (5000 to 10000), no turbulence model was activated in the numerical solver (laminar regime).

\subsection{Grid convergence analysis at $K C=6.3$}

The convergence of numerical results with increased grid resolution was checked with three different grids, labeled Grid1, Grid2 and Grid3 for the particular value $K C=6.3$, with little loss of generality. In the immediate vicinity of the plate, the spatial resolution of all grids is similar; hence, the convergence criterion that Courant numbers should always be less than 0.1 is satisfied with time steps of the order of $0.001 \mathrm{~s}$. In Grid 2 and Grid3, however, some stretching of the cells in the horizontal direction was implemented near the ends of the domain. The main features of these grids are given in Table 1. The symbols $\Delta x$ and $\Delta y$ represent the spatial mesh size in the horizontal and vertical directions, respectively. An aspect ratio $\Delta x / \Delta y$ equal to 2 used uniformly in Grid1 is considered to be very good as long as highly nonlinear phenomena such as wave breaking do not occur (See Table 1 of Jacobsen et. al 2011b).

The first numerical experiments were carried out without vertical plate to establish the location of the nodes in the wave field, and to estimate the variation and amplitudes of the horizontal and vertical velocities at the intended location of the plate. 
Table 1 - Mesh characteristics of 2-D numerical grids

\begin{tabular}{|c|c|c|c|c|c|}
\hline & \multicolumn{2}{|c|}{ Vicinity of plate } & \multicolumn{2}{c|}{$\begin{array}{c}\text { Vicinity of vertical } \\
\text { boundaries }\end{array}$} & \multirow{2}{*}{ Number of cells in domain } \\
\cline { 2 - 5 } & $\Delta y(\mathrm{~m})$ & $\Delta x / \Delta y$ & $\Delta y(\mathrm{~m})$ & $\Delta x / \Delta y$ & \\
\hline Grid1 & 0.004 & 2 & 0.004 & 2 & $\approx 570000$ \\
\hline Grid2 & 0.004 & 2 & 0.004 & 8 & $\approx 285000$ \\
\hline Grid3 & 0.004 & 2 & 0.004 & 20 & $\approx 140000$ \\
\hline
\end{tabular}

Figure 1 shows free surface profiles at intervals of approximately 1/25 of the wave period with or without the plate. Jacobsen (2011a), presents similar results (without any plate) in Figure 4.5 of his $\mathrm{PhD}$ dissertation for a linear standing wave. Due to the nonlinearity of the wave, the crests in Figure 1 are further from the still-water line than the troughs, i.e. outside the wave relaxation region. The occurrence of a wave node is clear at $x \approx 13.3 \mathrm{~m}$ (or about 2.75 wavelengths from the inlet), where the plate was later added to the numerical grid. Figure 1 indicates that there is no noticeable change in the location of the nodes when the plate is added. Figure 2 compares the velocity vectors, vorticity fields normalized by the circular frequency of the wave and free surface profiles at the location of the plate with or without the plate. Subfigures (a) to (h) (no plate) in Figure 2 are approximately an eighth of a wave period apart and correspond to subfigures (i) to (p) (plate in position). In subfigures (a) to (d), as the free surface rotates clockwise, the velocity field first strengthens and then weakens. In subfigure (e), the direction of rotation of the free surface and the velocity vectors are reversed and the cycle is later repeated. Subfigures (i) to (p) show vortex shedding from the plate and how the vortices travel in the velocity field surrounding the plate. 
Figure 3 shows horizontal and vertical velocities at a point $0.25 \mathrm{~m}$ below the free surface and one node away from the plate. After about 25 seconds, a stationary wave field is established with the occurrence of harmonic horizontal velocities and sub-harmonic vertical velocities, as noted in Keulegan and Carpenter (1958). Vertical components are relatively small compared, i.e. less than $3 \%$ of their horizontal counterparts in relative terms. A least-square fit of the horizontal velocity calculations to the function $U_{m} \cos \{(2 \pi / T) t\}$ was performed using the MATLAB $^{\circledR}$ function $f i t$, and is also shown in

Figure 3. There is an excellent agreement between numerical data and sinusoidal fit (goodness of fit measure, $R^{2}=0.99$ ), as in all calculations, and the parameter $U_{m}$ represents an accurate estimate of the amplitude of oscillating horizontal velocities.

In oscillating flows, the total horizontal force $F$ per unit width acting on the vertical plate can be decomposed into an inertial term and a viscous term, as in Morison's equation: (Keulegan and Carpenter, 1958):

$$
F=\rho C_{m} A_{o} \frac{d U}{d t}+\frac{1}{2} \rho C_{d} D U|U|
$$

$U$ represents the horizontal velocity without the object, and $\rho$ the fluid density. $A_{o}$ is defined as an equivalent circular area equal to $\pi D^{2} / 4$. Substituting $U=-U_{m} \cos \{(2 \pi / T) t\}$ in Equation (1) and setting $\theta=(2 \pi / T) t$ yields:

$$
\frac{F}{\rho U_{m}^{2} D}=\frac{\pi^{2}}{2 K C} C_{m} \sin (\theta)-\frac{1}{2} C_{d}|\cos (\theta)| \cos (\theta)
$$


In the laboratory experiments of Keulegan and Carpenter (1958), the starting and ending times of the force cycle on the plate were found from the position of the free surface. Since drag and inertia coefficients are estimated from fitting the sum of terms containing periodic (trigonometric) functions to the force data, the starting and ending points of the force cycle directly affect the magnitude of the coefficients. The starting time of the force cycle is chosen when the upwardly surging free surface (on the left of the plate) reaches the mean still water position, and the ending time is when the same position is reached after one period.

The procedure to estimate the starting and ending times of the force cycle in the OpenFOAM numerical experiments is as follows. From the instantaneous free surface elevation $\eta$, the integral $I_{x}=\int \eta^{2} d x$ is estimated between the two antinodes closest to the plate, and plotted as in Figure 4(a). The minima of this quantity indicate the times at which the overall free surface deflection is zero or very small. The times at which these minima are located are then transferred to the horizontal force data shown in Figure 4 (b), where the same normalization as in Equation (2) has been applied. In Figure 4(b), for example, Point $\mathrm{A}$ is an instant when the free surface is surging upwards on the left of the plate, and Point B denotes the next such event after one period.

The OpenFOAM CFD solver calculates the fluid velocities and pressure fields in the numerical domain for the given initial and boundary conditions. The dynamic pressure on the surface of the plate obtained from the CFD solver was integrated to assess the force on the plate. The MATLAB fit function was used again to estimate the coefficients in Equation (2), $C_{m}$ and $C_{d}$,from the normalized force data. Figure 5 shows these hydrodynamic coefficients from the 3 grids - Grid1, Grid2 and Grid3 - at $K C=6.3$. 


\begin{abstract}
Although the number of cells decreases fourfold from Grid1 to Grid3, there is no significant difference in the magnitude of the coefficients, suggesting that results have effectively converged with the highest-resolution Grid1. Unless specified otherwise, results presented in the next section are derived with Grid1.
\end{abstract}

Although the present calculations are concerned with a more restricted two-dimensional domain, it was always necessary to allow for the establishment of the imposed stationary wave field (about 25 seconds of simulation time), while a choice was also made to consider a sufficient number of force cycles afterwards (for an additional simulation time of about $35 \mathrm{~s}$ ) to ascertain the stability of the results. Therefore, specific runs on an 8 processor system typically took 3 to 4 days with the selected 570000 cell grid (Grid1).

\title{
2.4 Results and discussion
}

In an extensive review of the hydrodynamic forces associated with plates in oscillating flows, Dalzell (1978) presented drag and inertia coefficients from multiple sources, including Keulegan and Carpenter (1958). Dalzell (1978) suggested that when the average amplitudes of the inertia and drag force components per unit width, defined in Equation (1), are normalized by $\rho \omega^{2} D^{3}$, where $\omega$ is the circular frequency $2 \pi / T$, they trend closely as proportional to $(A / D)^{1.5}$, where $A$ is the amplitude of the oscillatory flow. Noting that $K C=2 \pi(A / D)$ and using the least-square-fit proportionality coefficients proposed by Dalzell (1978), the corresponding approximations of $C_{d}$ and $C_{m}$ can be plotted along the data of Keulegan and Carpenter (1958) in Figure 6. Also shown are the corresponding OpenFOAM numerical simulations, which reproduce the data fairly well in spite of a very wide range for $K C$. In particular, an apparent change in inertia coefficient trend slopes at $K C$ values in the neighborhood of 15 is well captured in the 
calculations. Keulegan and Carpenter (1958) observed that for the largest values of $K C$, the drag coefficient seemed to reach the well-known uniform-flow limit of about 2 (Stanton, 1903; Fage and Johansen, 1927). Their parametric range for $K C$ exceeded 100, however, a limit for which numerical simulations were not attempted. Such high KC numbers could be obtained by subjecting the plate to larger velocities, corresponding to higher (standing) waves. The behavior of such waves and their potential breaking in the relaxation zone (near the wave generator) would have to be assessed. Another way could be to use shorter plates, but those would perhaps span too few grid cells and lead to numerical instabilities.

\section{Flow from submerged oscillating three-dimensional plates}

For many small-scale (point) wave energy converters deployed in deep water, the production of electrical power from the incident wave field relies on the relative motion between a floating body at the free surface and a submerged body underneath ( $\mathrm{Yu}$ and $\mathrm{Li}$, 2013). The power take-off mechanism generally is an integral part of the connection, as it exerts a resistive damping force. To optimize relative body motion, it is essential to design the submerged component with sufficiently large inertia and drag coefficients. Horizontal heaving circular disks are often selected to this end. Circular disks and square plates have also been used in the offshore oil and gas industry to reduce the resonant oscillations of deep water spar platforms. Although such bodies have very simple geometries, experimental and computational work continues today to better understand their hydrodynamic behavior, especially in nonlinear conditions, at high KeuleganCarpenter numbers; the effects of high frequencies of oscillation, body porosity and proximity to boundaries also warrant special consideration. A brief literature review is 
proposed below, starting with the definition of basic terminology, before specific OpenFOAM simulations are set up. Results are presented and discussed afterwards.

\subsection{Literature review}

First, important dimensionless parameters in the analysis of oscillating disks and plates are defined. The Keulegan-Carpenter number $K C=2 \pi(A / D)$ was introduced earlier, but here, $A$ denotes the amplitude of forced body oscillations rather than that of ambient fluid oscillations. Thus, $K C$ is a measure of nonlinearity associated with body motions of finite magnitude, and the limit $K C \rightarrow 0$ corresponds to linearized (small motion) hydrodynamics. The frequency parameter $\beta=f D^{2} / v$ is often used in lieu of the betterknown Reynolds number $R e$, where $f$ denotes the frequency of oscillation and $v$ the kinematic viscosity of the fluid. $\beta$, as well as $\operatorname{Re}=(K C) \beta$, is a measure of nonlinearity associated with fluid viscous effects, including those arising from eddy formation (turbulence), and the limit $\beta \rightarrow \infty$ corresponds to inviscid hydrodynamics. Vanishing $K C$ numbers combined with large frequency parameters would define the range of validity of linearized potential theory, which remains a benchmark for field-scale hydrodynamics.

In an unbounded fluid domain, the hydrodynamic force acting on three-dimensional oscillating bodies can be modeled using a generalization of Morison's equation, given earlier as Equation (1) in a two-dimensional context. Thus, the instantaneous fluid force $F$ can be written as:

$$
F=\rho C_{m} V \frac{d U}{d t}+\frac{1}{2} \rho C_{d} S U|U|
$$


In Equation (3), $S$ is the surface area of the object perpendicular to the direction of motion, i.e. $\pi D^{2} / 4$ for a circular disk and $D^{2}$ for a square plate; in such cases, the immersed volume $V$ simply is equal to the product of $S$ by the thickness of the plate. Following Keulegan and Carpenter (1958), the drag and inertia coefficients can be determined as follow from the force time history, when the instantaneous velocity is of the form $U=A \omega \cos (\omega t)=U_{m} \cos \theta$.

$$
\begin{aligned}
& C_{d}=-\frac{3}{4 \rho S U_{m}^{2}} \int_{0}^{2 \pi} F(\theta) \cos (\theta) d \theta \\
& C_{m}=\frac{1}{\pi \rho V U_{m} \omega} \int_{0}^{2 \pi} F(\theta) \sin (\theta) d \theta
\end{aligned}
$$

We remark here that the notation $C_{a}$ is traditionally preferred over $C_{m}$ for forced body oscillations in an otherwise calm fluid; when instead an oscillatory flow is generated $a$ priori, as in the experiments of Keulegan and Carpenter (1958), the force component associated with inertia is larger since it also includes the effect of the a priori pressure field (the Froude-Krylov force in potential theory terminology). Moreover, it is common to linearize the drag term in Equation (3) as $B U$. The value of $B$ that best represents the quadratic form over a period of oscillation is $B=4 \rho C_{d} S U_{m} /(3 \pi)$. If the oscillating object is close enough to the free surface (shallow submergence) and generates waves that are allowed to propagate away, an additional energy dissipation mechanism is available, aside from viscous effects. In such cases, $B$ would also include this contribution, which at vanishing $K C$ numbers would be the well-known (radiation wave) damping coefficient of 
potential theory. This subtle point will be used later to assess the accuracy of OpenFOAM simulations

The potential solution for the added mass of a thin disk oscillating along its axis in an unbounded fluid was determined a long time ago by Lamb (1932). The result, $m_{a}=\rho D^{3} / 3$, can be used to non-dimensionalize the mass term in Equation (3) when studying so-called heave plates. Hence, the inertia behavior of such simple shapes is often presented in terms of $A^{\prime}=\left(\rho C_{m} V\right) / m_{a}$. The expected limit of $A^{\prime}$ when $K C$ tends to 0 is 1 for deeply submerged configurations. Similarly, the behavior of $C_{d}$ is not well defined when $K C$ approaches zero (He et. al, 2007), so that the coefficient $C_{b}=(K C) C_{d} /(4 \pi)$ has been used extensively instead in the literature (e.g., Lake et al., 2000; Vu et al., 2004; He et. al, 2007; Tao and Dray, 2008; Wadhwa et al., 2010; Garrido-Mendoza et al., 2013). The expression for $C_{b}$ can be recovered by non-dimensionalizing $B$ with $2 \omega m_{a}$ for circular disks.

Dalzell (1978) reviewed existing data for three-dimensional square plates and circular disks as well. Just as in the case of 2-D plates, he noted that the average amplitude of the drag force component, defined in Equation (3) and normalized here by $\rho \omega^{2} D^{4}$, was nearly proportional to $(A / D)^{1.5}$ for large enough amplitude ratios, as shown in his Figure 10. This observation (and the coefficient of proportionality he suggests) can be translated into the curve fit $C_{b} \approx 0.59 K C^{0.5}$ for $K C$ numbers larger than about one.

Minamizawa and Endoh (1984) carried out an important experimental investigation of oscillating disks over a broad range of Reynolds and $K C$ numbers. Their measurements of drag coefficients, reproduced here in Figure 7, show that in all generality, $C_{d}$ depends on both $K C$ and $\operatorname{Re}$ (for which the amplitude ratio, in the original paper, and $2 \pi \beta$ are proxies, 
Table 2 - Characteristics of recent experimental work on oscillating 3-D heave plates

\begin{tabular}{|c|c|c|c|c|c|}
\hline Reference & Shape $^{\mathrm{a}}$ & $\zeta$ & Frequency $(\mathrm{Hz})$ & $K C$ & $\beta^{\mathrm{b}} \times 10^{-3}$ \\
\hline Prislin et al. $(1998)$ & $\mathrm{S}$ & $1 / 60$ & $1.4,2.0^{\mathrm{c}}$ & $0.1-2.4$ & $47^{\mathrm{c}}-410$ \\
\hline Lake et al. $(2000)$ & $\mathrm{C}$ & $1 / 24$ & 3.91 & $0.08-0.4$ & 128 \\
\hline Vu et al. $(2004)$ & $\mathrm{C}$ & $1 / 100$ & $0.9,1.2$ & $0.04-1.6$ & 36,48 \\
\hline Chua et al. $(2005)$ & $\mathrm{S}$ & $1 / 40$ & $0.5,1.0$ & $0.2-1.3$ & 70,140 \\
\hline He et al. $(2007)$ & $\mathrm{C}$ & $1 / 87.5-1 / 25$ & 4.0 & $0.01-1.1$ & 124 \\
\hline Tao and Dray $(2008)$ & $\mathrm{C}$ & $1 / 50$ & $0.1-1.0$ & $0.2-1.2$ & $16-160$ \\
\hline Wadhwa et al. $(2010)$ & $\mathrm{C}$ & $1 / 100$ & $1.0,1.8$ & $0.26-1.77$ & $40^{\mathrm{d}}$ \\
\hline
\end{tabular}

${ }^{\mathrm{a}} \mathrm{S}$ is for square plates, $\mathrm{C}$ for circular disks.

${ }^{\mathrm{b}}$ Rounded values.

c This value corresponds to oscillations in air.

${ }^{\mathrm{d}}$ Value based on a frequency of $1.0 \mathrm{~Hz}$ and kinematic viscosity of $10^{-6} \mathrm{~m}^{2} \mathrm{~s}^{-1}$. 
exhaustive, but it will be discussed in more details later, when comparing the data to Open FOAM simulations in Section 3.3. Specific motivations for these studies varied somewhat, even though underlying practical goals in the engineering context of offshore structures remained the potentials of heave plates to provide for additional motion damping, and for better natural-frequency tuning from additional added mass. Prislin et al. (1999) investigated single oscillating square plates as well as multiple-plate configurations. They concluded that drag damping at high Reynolds numbers becomes independent of $R e$, a finding essentially similar to that of Minamizawa and Endoh (1984). Lake et al. (2000) were concerned with Tension Leg Platforms (TLPs), and therefore analyzed not only single oscillating circular disks situated well below the sea surface, but similar disks fitted on vertical surface-piercing cylindrical columns, as in the earlier investigation of Thiagarajan and Troesch (1998), or in the later work of Tao and Thiagarajan (2003a, 2003b). The thickness ratio of the disks $\zeta$, defined as plate thickness over diameter, was relatively high in their experiments, at 1/24. By comparison with earlier results obtained with thinner disks (e.g. as documented in Dalzell, 1978), the authors noted that their measured drag coefficients were significantly lower. This later prompted a specific study of the effect of $\zeta$ conducted by He et al. (2007). The work of Lake et al. (2000) was confirmed, and as $\zeta$ was varied from $1 / 87.5$ to $1 / 25$, a notable reduction in drag was observed for the thicker disks, especially at $K C$ lower than about 0.4. Molin (2001) published a theoretical study investigating how the porosity of periodically arranged arrays of circular heave plates affect inertia and drag coefficients. It is based on potential theory, with porosity represented by a continuous parameter $\tau$, from 0 (solid disks) to 1 (free flow), modulating a heuristic boundary condition for the pressure 
drop across the disks. Results for single disks correspond to large (theoretically infinite) array spacing. Molin (2001) found that damping is non zero when both $K C$ and $\tau$ are non zero, and hence, reaches a maximum for some $K C$ and $\tau$ combination. The added mass coefficient, however, exhibits a discontinuous behavior when $\tau$ approaches zero: the solid disk result, corresponding to an added mass equal to $m_{a}$, is recovered for $\tau=0$ and any value of $K C$, but for any non zero porosity, the added mass coefficient tends to zero with $K C$. Understandably, Molin's work spurred several experimental investigations. Using small circular perforations to mimic positive values of $\tau$, Vu et al. (2004) qualitatively confirmed the damping behavior of porous oscillating disks inferred by Molin (2001). Tao and Dray (2008) later conducted a comprehensive series of tests on perforated circular disks as well, using a planar motion mechanism (PMM) to drive the disk oscillations. In their study, $\tau$ varied from 0 to 0.2 . Inertia and drag coefficients were obtained for $K C$ between 0.2 and 1.2, and oscillation frequencies of $0.1,0.5$ and $1.0 \mathrm{~Hz}$. In other experiments concerned with heave plate perforations, Chua et al. (2005) considered the hydrodynamic behavior of a $0.4 \mathrm{~m}$ wide, $0.01 \mathrm{~m}$ thick oscillating square plate with or without a single central opening (rather than distributed holes).

A more recent experimental study by Wadhwa et al. 2010 was concerned with the effect of the proximity of a solid boundary (seabed) on heaving circular disks. They concluded that a distance of about one diameter from the seabed corresponds to unbounded-fluid cases for the range of $K C$ under consideration. Interestingly, Garrido-Mendoza et al. (2013) used the measurements of Wadhwa et al. (2010) as a benchmark for numerical simulations based on the CFD package OpenFOAM. Therefore, both data and simulations from these two teams can be used to evaluate the performance of our 
numerical wave tank. In a follow-up study that also included experiments reminiscent of Lake et al.'s (2000), Garrido-Mendoza (2014) examined the hydrodynamic coefficients of a surface-piercing cylindrical column fitted with a circular heave plate, for various depths of submergence.

\subsection{Numerical experiments with OpenFOAM}

Given the numerical intensity of three-dimensional CFD simulations and the wide scope of published results, we deliberately focused our numerical experiments on basic cases that lend themselves well to the validation of a numerical wave tank. Since simultaneous Reynolds and Froude scalings are not experimentally possible and that gravity effects are strong in field-scale free-surface hydrodynamics, it is important to make sure that laboratory-scale experimental results are not very sensitive to $R e$. This can be practically achieved, as is clear, for example, from Figure 7, where drag coefficients are shown to level off at sufficiently high frequency parameters. The range of experimental frequency parameters and $K C$ numbers in Table 2 represents values that may be considered adequate for extrapolation from model-basin scale to field scale.

The main set of numerical simulations closely follows the experimental matrix of Tao and Dray (2008), for thin oscillating solid circular disks (the effect of disk porosity is not investigated). Thus, computations were carried out at three oscillation frequencies $(0.1$ $\mathrm{Hz}, 0.5 \mathrm{~Hz}$ and $1.0 \mathrm{~Hz}$ ) and the original $K C$ range of 0.2 to 1.2 , at intervals of 0.2 ; a low value $K C=0.03$ was added to test the $K C \rightarrow 0$ limit. The diameter of the circular disk is $0.4 \mathrm{~m}$ and its thickness $0.008 \mathrm{~m}$. Additional computations were undertaken for the $1 \mathrm{~Hz}$ case, but with the disk brought up in closer proximity to the free surface (shallow submergence of $0.2 \mathrm{~m}$ ); this was intended, in particular, to test the ability of OpenFOAM 
to resolve wave damping and added mass characteristics that would be predicted by potential theory in the $K C \rightarrow 0$ limit.

The effect of disk thickness was investigated too using a disk thickness ratio $\zeta$ equal to $1 / 25$ and an oscillation frequency of $4 \mathrm{~Hz}$, for $K C$ between 0.05 and 0.9 . To this end, a smaller disk diameter was used in the numerical wave tank $(D=0.177 \mathrm{~m})$ with essentially the same thickness, so that results would be comparable to the laboratory measurements of He et al. (2008).

The case of a square plate with a width of $0.4 \mathrm{~m}$ and thickness of $0.01 \mathrm{~m}$ was considered as well, at frequencies of $0.5 \mathrm{~Hz}$ and $1.0 \mathrm{~Hz}$ and for a $K C$ range from 0.1 to 1.3. The setup corresponds to the experimental data of Chua et al. (2005).

The OpenFOAM CFD solver used for modeling the oscillating disk problem is called waveDyMFoam. It is a modified version of the standard interDyMFoam solver that includes the wave generation tool box developed by Jacobsen et al. (2011b). It differs from the solver in Section 2 as it allows the computational mesh to be dynamically deformed. Details regarding the dynamic mesh motion capabilities can be found in Jasak and Tuković (2004), and Jasak and Tuković (2010). Higuera et. al (2015) also briefly broach this topic while discussing paddle-type numerical wave makers. In the waveDyMFoam solver as used in this study, the motion of the plate, which forms the moving boundary, is prescribed, meaning that the velocity and displacement of the plate are known. A Laplace equation is now solved numerically to calculate the deformation velocity $\mathbf{U}_{\mathbf{p}}$ of the points that make up the computational grid, as shown below,

$$
\nabla \bullet\left(\gamma \nabla \mathrm{U}_{\mathrm{p}}\right)=0
$$


In Equation (6), $\gamma$ locally equals the inverse of the distance of a given point from the moving boundary, which ensures that points close to the plate move the most and vice versa. The motion of the plate forms the boundary condition for the solution of Equation (6). Once the deformation velocities of the points are calculated, their new locations can be easily calculated as:

$$
\mathbf{X}_{\text {new }}=\mathbf{X}_{\text {old }}+\mathbf{U} \mathbf{p} \Delta \mathrm{t}
$$

where $\mathbf{X}_{\text {old }}$ and $\mathbf{X}_{\text {new }}$ are the position vectors of the point before and after mesh deformation and $\Delta t$ denote the time step of the computation. For a static mesh (as in Section 2, for example), the application of the Reynolds transport theorem to the computational cells is simpler since their boundaries are fixed. In the case of a dynamic mesh (as in waveDyMFoam), the transport theorem is modified by the velocities of the cell boundaries (cf. Equations (1) to (4) in Jasak and Tuković (2004)), which are derived from the mesh deformation velocities in Equation (6).

The solver computes the volume of fluid fraction, pressure and velocities. If turbulence modeling is activated, turbulence quantities such as turbulent kinetic energy, $k$, and turbulence dissipation rate, $\omega$ are also computed. In this study, the $k-\omega$ turbulence model described in Jacobsen et al. (2011b) is activated whenever Re is above or near 10000. Although it is not available in the default OpenFOAM library (version 2.1.0), we also implemented and tested the low Reynolds number $k$ - $\omega$ model of Wilcox (2008). No noticeable differences in the results were found, however. This was attributed to the type of geometry considered here, where flow separation takes place at the plate edges due to 
the presence of sharp corners; for blunt cylinders, the turbulence model must be able to correctly predict the flow separation point instead.

When the plate is deeply submerged, i.e., practically, at depths greater than $D$ for the range of $K C$ under consideration, surface-wave effects become negligible, and the total force on the disk is composed of viscous drag and inertia only. This allows smaller computational domains. With plates located $0.6 \mathrm{~m}$ below the free surface, the radial boundary of the circular computational domain was $3.5 \mathrm{~m}$ from the center and its depth $1.675 \mathrm{~m}$, as in Tao and Dray (2008).

A convenient length scale for choosing the size of cells in the computational domain is the thickness of the thin circular disks tested by Tao and Dray (2008), i.e. $0.008 \mathrm{~m}$. A detail of a radial cross section across the numerical wave tank is shown in Figure 8 for the selected 3-D mesh, which consists of 569200 cells; cell vertices are red lozenges and the blue area represents a portion of a disk. Grid convergence tests were performed at $K C$ $=1.2$ for an oscillation frequency of $1 \mathrm{~Hz}$. It may be noted that in this case, the amplitude of oscillations is about 10 times the disk thickness. In defining coarser grids, cells were stretched by factors of 2 either in a radial direction away from the edge of the disk, or in the vertical direction on either side of the disk. Figure 9 demonstrates that the hydrodynamic coefficients $C_{b}$ and $A$ ' have converged well for the selected grid. In the two pairs of curves, the lower ones correspond to vertically coarser grids, suggesting a greater sensitivity of the simulations to vertical mesh resolution.

The quality of the computational mesh can be checked and quantified by measures such as aspect ratio, mesh non-orthogonality and skewness. These measures can be obtained using the OpenFOAM mesh utility called checkMesh. The definition of these mesh- 
quality measures and options for running the mesh utility can be found at https://openfoamwiki.net/index.php/CheckMesh. Sieger et. al (2015), for example, use this utility for an automobile simulation. Table (3) presents results from checkMesh for the present study, when the mesh deformation is zero and when it is maximal. Note that only cells close to the plate (bounding box with diagonal vertices $\{-0.3,-0.7,-0.3\}$ and $\{0.3,0.5,0.3\})$ were considered since the mesh is intentionally stretched away from the plate.

Table 3 - Mesh quality measures from OpenFOAM checkMesh utility

\begin{tabular}{|l|l|l|l|}
\hline & $\begin{array}{l}\text { Maximun } \\
\text { Aspect ratio }\end{array}$ & $\begin{array}{l}\text { Average } \\
\text { Mesh non-orthogonality }\end{array}$ & $\begin{array}{l}\text { Maximum } \\
\text { Skewness }\end{array}$ \\
\hline Before deformation & 6 & 6.4 & 0.98 \\
\hline Maximal deformation & 8 & 6.8 & 0.98 \\
\hline
\end{tabular}

The values shown for all mesh quality measures are within an acceptable range. (Sieger et. al (2015)) .

In the case of shallow disk-submergence simulations, the numerical domain was extended to $9.5 \mathrm{~m}$ from the center of the grid, so that there would be enough time to record several force cycles before outgoing waves reach the outer boundary. This corresponds to about 1 million cells. For these tests, the disk was placed $0.2 \mathrm{~m}$ (half a diameter) below the free surface. The frequency of oscillation was restricted to $1 \mathrm{~Hz}$ since a lower frequency would have required a larger numerical domain and greater computational resources to accommodate longer, faster waves. Using the dispersion relation, the wavelength of the waves generated by the disk oscillating at $1 \mathrm{~Hz}$ is estimated to be $1.5 \mathrm{~m}$. Since transients 
in this forced-oscillation setup are very short, about 6 periods of force data were therefore available.

The effect of a high plate thickness ratio $(\zeta=1 / 25)$ was also investigated, as in He et al. (2008), with a focus on very low $K C$. In such cases, the vortices shed from the edge of the plate are small, and comparable in size to the plate thickness. $\zeta$ was increased from previous simulations by reducing the disk diameter to $0.1778 \mathrm{~m}$. Plate submergence then is about 3.5 diameters under the free surface, at a frequency of oscillation of $4 \mathrm{~Hz}$. The overall dimensions of the numerical grid are similar to those in previous cases of deeply submerged disks. In order to resolve the small vortical structures generated at the edges, however, especially in the low $K C$ range, a refined grid with six cells resolving the plate thickness is used. The total number of cells in this grid is now roughly one million. Some of the computations were also carried out on a coarser grid with only four cells resolving the plate thickness. Figure 10 shows a detail of the radial cross section for the highestresolution grid in these simulations, as well as an overall view of the mesh over the disk. Calculations for single numerical experiments on an 8 processor system typically varied between 1 and 2 days at higher oscillating frequencies (e.g., $1 \mathrm{~Hz}$ ), and between 3 and 5 days at lower oscillating frequencies (e.g., $0.1 \mathrm{~Hz}$ ) for grids of about 550000 cells. Flow establishment (transient behavior from initial conditions) was here extremely fast (essentially zero). Overall simulation times were extended to about $6 \mathrm{~s}$ (at 1Hz) to $40 \mathrm{~s}$ (at $0.1 \mathrm{~Hz}$ ) to ensure stable conditions.

\subsection{Results and discussion}

Figure 11 shows free surface waves radiating from the oscillating disk after about 6 cycles of oscillation of the disk, when it (the disk) is close to the surface. The $K C$ number 
is 1.2. The free surface deflections have been normalized by the amplitude of oscillation of the disk. The lateral size of the domain is large compared to the size of the disk. Near the disk, the magnitude of deflection of the free surface is similar to the amplitude of oscillation of the disk.

As in section 2.3, the dynamic pressure field obtained from the OpenFOAM solver was integrated over the surface of the disk to obtain the force, and the corresponding force coefficients could then be estimated (See for example, Equations 4 and 5). Figure 12 shows selected experimental data (markers), and OpenFOAM numerical simulations (lines) for the reduced drag coefficient $C_{b}$ of thin oscillating circular disks as a function of $K C$; numbers in the legend refer to the frequency parameter $\beta$. The three sets of OpenFOAM simulations for the deeply submerged disk (blue, green and red continuous lines) representative of the experimental setup of Tao and Dray (2008) show little dependence on $\beta$. This confirms the findings of Minamizawa and Endoh (1984) for high frequency parameters, as is clear in Figure 7. Moreover, any dependence of $C_{b}$ on $\beta$, at a given $K C$, should be such that drag decreases with $\beta$ (or equivalently, with $R e$ ). In this respect, the data of Tao and Dray (2008) is puzzling since it shows significant scatter between results for the three selected values of $\beta$ (with oscillation frequencies of $0.1,0.5$ and $1 \mathrm{~Hz}$ ), while the highest drag coefficients correspond to the highest frequency parameter $(\beta=160000)$. The recently published OpenFOAM simulations of GarridoMendoza et al. (2013), shown here as a dashed red line, are significantly offset upward from our own calculations as well as from the data of Wadhwa et al. (2010) which they aimed to reproduce. The cause for such apparent discrepancies is not obvious, but we 
note that Garrido-Mendoza et al. $(2013,2014)$ did not include any turbulence model in their solver (laminar regime).

The convergence of $C_{b}$ to zero as $K C$ vanishes constitutes an accuracy criterion for either measurements or numerical simulations. As pointed out in Lake et al. (2000), the attribution of a non-zero offset to friction is not very credible for thin circular disks oscillating along their axis, since the wetted area resisting the flow is negligible. In this respect, some of the experimental data as well the simulations of Garrido-Mendoza et al. (2013) do not seem satisfactory, and $C_{b}$ offsets of the order of 0.1 as $K C \rightarrow 0$ suggest accuracy problems with those particular measurements and numerical simulations. Our OpenFOAM simulations of $C_{b}$ for the deeply submerged disk do not converge to zero with $K C$ either, but offsets are much smaller $(0.04$ to 0.05 at $K C=0.03)$ and compare well with the best measurements.

The light-purple continuous line in Figure 12 corresponds to OpenFOAM simulations for a shallow disk oscillating at $1 \mathrm{~Hz}$, as described in Section 3.2. When $K C$ vanishes, the residual value of $C_{b}$ should correspond to wave radiation damping in the small-motion limit of linear potential theory, which was separately calculated as 0.0675 using the deepwater algorithm of Martin and Farina (1997). Because the corresponding deep-disk calculations of $C_{b}$ (red continuous line) do not converge perfectly to zero with $K C$, the ability of OpenFOAM to reproduce wave radiation damping for shallow disk submergences was assessed by considering the difference between the $K C \rightarrow 0$ limits (i.e., at $K C=0.03$ ) for shallow and deep submergence cases, respectively. This approach implies that damping from viscous effects and turbulent eddy formation, as modeled in the numerical wave tank, is essentially the same at low $K C$ values whether the disk is 0.6 
$\mathrm{m}$ or $0.2 \mathrm{~m}$ below the free surface. This would yield a residual of 0.0889 for $C_{b}$, in fair agreement with the estimate from potential theory.

The range of $K C$ considered here, of order one or less, is too low to verify the proportionality of $C_{b}$ to the square root of $K C$ suggested by Dalzell (1978) or Minamizawa and Endoh (1984). Instead, the behavior of $C_{b}$ seems to be quasi linear in Figure 12. He et al. (2007) argued that the steeper slope they observed, for $K C$ less than about 0.15 in Figure 12 (thin disk with $\zeta=1 / 87.5$ ), would correspond to the reinforcing interaction of vortices generated near the sharp corners, as they roll along the disk's edge (Mode 3). At higher $K C$, vortex interaction would result instead in asymmetric vortex generation with the unidirectional shedding of vortex pairs (Mode 4). Neither the other data nor the simulations displayed in Figure 12 suggest the existence of a distinct flow regime that would correspond to He et al. (2007)'s Mode 3, although they may simply be too sparse to resolve He et al. (2007)'s measured local feature (steeper slope) at low $K C$. Figure 13 shows the reduced inertia coefficient $A^{\prime}$ for the component of the hydrodynamic force in phase with disk acceleration ${ }^{1}$. The conventions adopted for the display of experimental data and simulations are identical to those in Figure 12. The measurements of Wadhwa et al. (2010), or the processing thereof to determine $A$ ', are questionable since the $K C \rightarrow 0$ limit for $A$ ' is 1 by definition in an unbounded fluid (i.e., practically, for deeply submerged disks far away from solid boundaries). All other results for deeply submerged disks are generally following the same trend as $K C$ varies; among them, experimental data are more scattered and not available at $K C$ less than 0.2. Numerical simulations miss the zero $K C$ limit upward by about 0.1 . Here again, the

\footnotetext{
${ }^{1}$ The added-mass coefficient $\left(A^{\prime}\right)$ data of Vu et al. (2004) is reproduced from - and referenced as in Wadhwa et al. (2010), although the quotation is incorrect.
} 
markedly different results for a shallower disk are 0.0506 higher at $K C=0.03$ than their deep submergence counterparts. This compares well with the value $A^{\prime}=1.0463$ (differential of 0.0463 ) determined from the potential theory algorithm of Martin and Farina (1997).

Figure 14 shows the experimental data of Lake et al. (2000) and He et al. (2007) for the reduced drag coefficient $\left(C_{b}\right)$ of oscillating circular disks of various thickness ratios as a function of $K C$; numbers in the legend refer to $\zeta$. Also displayed are OpenFOAM numerical simulations. There is remarkable agreement between the two experimental data sets for the thicker disks ( $\zeta=1 / 24$ and $1 / 25$, respectively), with $K C$ less than 0.4 . According to He et al. (2007), the distinctly smaller slope for this setup at $K C<0.4$, as opposed to measurements at higher oscillation amplitudes or with thinner disks, would correspond to a lack of interaction among vortices generated at the disk's corners (Modes 1 and 2). At larger $K C$ or with smaller values of $\zeta$, vortex interaction would lead to two regimes (Modes 3 and 4) that were briefly discussed above in reference to Figure 12. OpenFOAM simulations of a thicker disk $(\zeta=1 / 25)$, including results corresponding to grids of different and higher resolutions in the vicinity of the disk, do capture the occurrence of relatively smaller values of $C_{b}$ in general, and a shallower slope of $C_{b}$ as a function of $K C$ when $K C<0.4$ for this specific setup. The change in slope is somewhat less pronounced, however, with a broader transitional range for $K C$. It should be noted here that the relatively good convergence of thick oscillating disks to zero at low $K C$, in spite of their greater wetted area in the direction of motion, should invalidate the attribution of non-zero $C_{b}$ offsets to friction in the $K C \rightarrow 0$ limit. 
In order to highlight purported differences in flow structures when $\zeta$ and $K C$ vary, simulations corresponding to $\zeta=1 / 25$ and $K C=0.05$ on one hand, and $\zeta=1 / 50$ and $K C$ $=0.2$ on the other hand, were considered. Such a choice would correspond to distinctly different vortex generation patterns according to He et al. (2008). For both cases, velocity vectors and vorticity fields normalized by the circular frequency of oscillation are displayed in 8 successive panels throughout a cycle. Vortices in pairs rotate in the same direction (same vorticity sign) and their size is comparable to disk thickness in Figure 15 $(\zeta=1 / 25$ and $K C=0.05)$. By contrast, vortices in pairs counter-rotate (opposite vorticity signs), generating a strong jet between them, and are typically larger than disk thickness in Figure $16(\zeta=1 / 50$ and $K C=0.2)$. These results somewhat corroborate the findings of He et al. (2008).

Finally, Figure 17 shows selected experimental data and OpenFOAM numerical simulations for the reduced drag coefficient $\left(C_{b}\right)$ of thin oscillating square plates as a function of $K C$; numbers in the legend refer to $\beta$. This represents the counterpart of Figure 12 for square plates instead of circular disks. Once more, the simulations are generally well behaved, with little dependence on the frequency parameter and the expected convergence of $C_{b}$ toward zero at small $K C$ appears reasonable.

\section{Conclusions}

A numerical wave tank was setup and tested using the open source CFD solver OpenFOAM ${ }^{\circledR}$. This preliminary work is meant to represent a first step in better understanding WEC behavior when fluid flow nonlinearities are deemed significant (large waves and motions, viscous effects, turbulence etc.). The present modeling effort 
focused on two-dimensional submerged flat plates in an oscillatory flow, or of threedimensional submerged circular and square plates undergoing prescribed oscillations. Such cases have been extensively studied in hydraulic laboratories, and thus provide a valuable validation reference. Additionally, they often represent important elements of more complex designs (e.g., heave plates) that may critically affect system resonance and energy dissipation. Overall, numerical simulations performed very well in predicting hydrodynamic forces, and thus inertia and drag coefficients, for a broad range of Keulegan-Carpenter numbers, i.e., when nonlinear conditions prevail. The effect of wave radiation damping could be discerned from viscous drag in shallow submergence tests, and testing plates of different thickness ratios in the NWT confirmed subtle vortex shedding trends at low $K C$.

Data generally exhibit more scatter than the calculations, and sometimes converge poorly to known small motion (linear) limits. In fact, some data sets occasionally follow contradictory trends. In this respect, the expectation that a well designed NWT can be an invaluable complement to experimentation seems justified. The information gathered in simpler NWT studies, such as those presented here, can also be used as an input into more numerically efficient hydrodynamic models of intermediate complexity without requiring excessive computational resources.

\section{ACKNOWLEDGEMENTS}

This research was funded by a grant from the U.S. Department of Energy through the Hawaii National Marine Renewable Energy Center (Hawaii Natural Energy Institute, University of Hawaii). 


\section{REFERENCES}

Babarit, A., Hals, J., Muliawan, M.J., Kurniawan, A., Moan, T., Krokstad, J., 2012. Numerical benchmarking study of a selection of wave energy converters. Renew. Energy 41, 44-63.

Cummins, W.E., 1962. The impulse response function and ship motions. David Taylor Model Basin, Bethesda, MD, U.S. Dep. of the Navy, Tech. Rep. No. DTNSDRC 1661, 9 pp.; available online at http://dome.mit.edu/handle/1721.3/49049 (accessed 02/26/15).

Chua, K.C., Clelland, D., Huang, S., Sworn, A., 2005. Model experiments of hydrodynamic forces on heave plates. Proc. $24^{\text {th }}$ Int. Conf. Offsh. Mech. Arct. Eng., OMAE2005, Halkidiki, Greece, Paper 67459, 6 pp.

Dalzell, J.F., 1978. Non-linear forces on oscillating plates: review and analysis of the literature. Report No. SIT-DL-78-9-2031, Stevens Inst. Tech., Hoboken, New Jersey, U.S.A., 110 pp.

Fage, A., Johansen, F.C., 1927. On the flow of air behind an inclined flat plate of infinite span. Br. Aeronaut. Res. Counc. Rep. Memo. 1104, 81-106.

Garrido-Mendoza, C.A., Souto-Iglesias, A., Thiagarajan, K.P., 2013. Numerical simulation of hydrodynamics of a circular disk oscillating near a seabed. Proc. $32^{\text {nd }}$ Int. Conf. Offsh. Mech. Arct. Eng., OMAE2013, Nantes, France, Paper 11072, 9 pp.

Garrido-Mendoza, C.A., Thiagarajan, K.P., Souto-Iglesias, A., Bouscasse, B., Colagrossi, A., 2014. Numerical investigation of the flow features around heave plates oscillating close to a free surface or seabed. Proc. $33^{\text {rd }}$ Int. Conf. Offsh. Mech. Arct. Eng., OMAE2014, San Francisco, U.S.A., Paper 23818, 11 pp. 
Gerritsen, H., Sutherland, J., 2011. Users guide to physical modelling and experimentation. Experience of the HYDRALAB network. IAHR.

He, H., Troesch, A.W., Perlin, M., 2007. Hydrodynamics of damping plates at small $K C$ numbers. Proc. IUTAM Symp. Fluid Struct. Interact. Ocean Eng., Hamburg, Germany, 93-104.

Higuera, P., Losada, I. J., Lara, J. L., 2015. Three-dimensional numerical wave generation with moving boundaries. Coastal Engineering, 101, 35-47.

Jacobsen, N. G., 2011. A full hydro- and morphodynamic description of breaker bar development. PhD Dissertation.

Jacobsen, N. G., Fuhrman, D. R., Fredsøe, J., 2011. A wave generation toolbox for the open-source CFD library. Int. J. Numer. Methods Fluids 70(9), 1073-1088.

Jasak, H., Tuković, Ž, 2004. Automatic mesh motion for the unstructured finite volume method. Elsevier Science.

(http://citeseerx.ist.psu.edu/viewdoc/download?doi=10.1.1.126.6838\&rep=rep1\&type=pdf).

Jasak, H., Tuković, Ž, 2010. Dynamic mesh handling in OpenFOAM applied to fluidstructure interaction simulations. Proc. V Eur. Conf. Comput. Fluid Dyn. ECCOMAS CFD 2010, Lisbon, Portugal, 19 pp. (available at http://congress.cimne.com/eccomas/cfd2010/papers/01178.pdf, 12/05/2014).

Keulegan, G.H., Carpenter, L.H., 1958. Forces on cylinders and plates in an oscillating fluid. J. Res. Natl. Bur. Stand. 60(5), 423-440.

Lake, M., He, H., Troesch, A.W., Perlin, M., Thiagarajan, K.P., 2000. Hydrodynamic coefficient estimation for TLP and spar structures. J. Offsh. Mech. Arct. Eng. 122, 118124.

Lamb, Sir H., 1932. Hydrodynamics, sixth ed. Cambridge University Press, U.K. 
Li, Y., Yu, Y. 2012. A synthesis of numerical methods for modeling wave energy converter-point absorbers. Renew. Sustain. Energy Rev. 16, 4352-4364.

Martin, P.A., Farina, L., 1997. Radiation of water waves by a heaving submerged horizontal disk. J. Fluid Mech. 337, 365-379.

Minamizawa, M., Endoh, K., 1984. Fluid resistance on a disk oscillating in a liquid at rest. J. Chem. Eng. Japan 17(2), 186-191.

Molin, B., 2001. On the added mass and damping of periodic arrays of fully or partially porous disks. J. Fluids Struct. 15, 275-290.

Molin, B., 2002. Hydrodynamique des Structures Offshore. TECHNIP, Paris, France, 615 pp. (in French).

Prislin, I., Blevins, R.D., Halkyard, J.E., 1998. Viscous damping and added mass of solid square plates. Proc. $17^{\text {th }}$ Int. Conf. Ocean Offsh. Mech. Arct. Eng., OMAE98, Lisbon, Portugal, Paper 0316, 7 pp.

Sarpkaya, T., Isaacson, M., 1981. Mechanics of Wave Forces on Offshore Structures. Van Nostrand Reinhold, New York, NY, 651 pp.

Sheng, W., Lewis, A., 2012. Assessment of wave energy extraction from seas: numerical validation. J. Energy Resour. Technol. 134, 041701-1-041701-8.

Sieger, D., Menzel, S., Botsch, M., 2015. On shape deformation techniques for simulation-based design optimization. New challenges in grid generation and adaptivity for scientific computing. Springer.

Stanton, T.E., 1903. On the resistance of plane surfaces in a uniform current of air. Minutes Proc. Inst. Civ. Eng. 156, 78-139. 
Tao, L., Thiagarajan, K.P., 2003a. Low $K C$ flow regimes of oscillating sharp edges, I: vortex shedding observation. App. Ocean Res. 25, 21-35.

Tao, L., Thiagarajan, K.P., 2003a. Low $K C$ flow regimes of oscillating sharp edges, II: hydrodynamic force coefficients. App. Ocean Res. 25, 53-62.

Tao, L., Dray, D., 2008. Hydrodynamic performance of solid and porous heave plates. Ocean Eng. 35, 1008-1014.

Thiagarajan, K.P., Troesch, A.W., 1998. Effects of appendages and small currents on the hydrodynamic heave damping of TLP columns. J. Offsh. Mech. Arct. Eng. 120, 37-42.

Vu, K.H., Chenu, B., Thiagarajan, K.P., 2004. Hydrodynamic damping due to porous plates. Proc. World Sci. Eng. Acad. Soc., WSEAS, Corfu, Greece, 5 pp (available at http://www.wseas.us/e-library/conferences/corfu2004/papers/488-360.pdf, 09/30/2014).

Wadhwa, H., Krishnamoorthy, B., Thiagarajan, K.P., 2010. Variation of heave added mass and damping near seabed. Proc. $33^{\text {rd }}$ Int. Conf. Offsh. Mech. Arct. Eng., OMAE2010, Shangai, China, Paper 20456, 7 pp.

Wilcox, D. C., 2008. Formulation of the k-omega turbulence model revisited. Am. Inst. Aeron. Astronaut. (AIAA) J. 46(11), 2823-2838.

Yu, Y., Li, Y. 2013. Reynolds-Averaged Navier-Stokes simulation of the heave performance of a two-body floating-point absorber wave energy system. Comput. Fluids 73, 104-114. 
Table 1 - Mesh characteristics of 2-D numerical grids

\begin{tabular}{|c|c|c|c|c|c|}
\hline & \multicolumn{2}{|c|}{ Vicinity of plate } & \multicolumn{2}{c|}{$\begin{array}{c}\text { Vicinity of vertical } \\
\text { boundaries }\end{array}$} & \multirow{2}{*}{ Number of cells in domain } \\
\cline { 2 - 5 } & $\Delta y(\mathrm{~m})$ & $\Delta x / \Delta y$ & $\Delta y(\mathrm{~m})$ & $\Delta x / \Delta y$ & \\
\hline Grid1 & 0.004 & 2 & 0.004 & 2 & $\approx 570000$ \\
\hline Grid2 & 0.004 & 2 & 0.004 & 8 & $\approx 285000$ \\
\hline Grid3 & 0.004 & 2 & 0.004 & 20 & $\approx 140000$ \\
\hline
\end{tabular}

Table 2 - Characteristics of recent experimental work on oscillating 3-D heave plates

\begin{tabular}{|c|c|c|c|c|c|}
\hline Reference & Shape $^{\mathrm{a}}$ & $\zeta$ & Frequency $(\mathrm{Hz})$ & $K C$ & $\beta^{\mathrm{b}} \times 10^{-3}$ \\
\hline Prislin et al. $(1998)$ & $\mathrm{S}$ & $1 / 60$ & $1.4,2.0^{\mathrm{c}}$ & $0.1-2.4$ & $47^{\ddagger}-410$ \\
\hline Lake et al. $(2000)$ & $\mathrm{C}$ & $1 / 24$ & 3.91 & $0.08-0.4$ & 128 \\
\hline Vu et al. (2004) & $\mathrm{C}$ & $1 / 100$ & $0.9,1.2$ & $0.04-1.6$ & 36,48 \\
\hline Chua et al. $(2005)$ & $\mathrm{S}$ & $1 / 40$ & $0.5,1.0$ & $0.2-1.3$ & 70,140 \\
\hline He et al. $(2007)$ & $\mathrm{C}$ & $1 / 87.5-1 / 25$ & 4.0 & $0.01-1.1$ & 124 \\
\hline Tao and Dray $(2008)$ & $\mathrm{C}$ & $1 / 50$ & $0.1-1.0$ & $0.2-1.2$ & $16-160$ \\
\hline Wadhwa et al. $(2010)$ & $\mathrm{C}$ & $1 / 100$ & $1.0,1.8$ & $0.26-1.77$ & $40^{\mathrm{d}}$ \\
\hline
\end{tabular}

${ }^{\text {a }} \mathrm{S}$ is for square plates, $\mathrm{C}$ for circular disks.

${ }^{\mathrm{b}}$ Rounded values.

c This value corresponds to oscillations in air.

${ }^{\mathrm{d}}$ Value based on a frequency of $1.0 \mathrm{~Hz}$ and kinematic viscosity of $10^{-6} \mathrm{~m}^{2} \mathrm{~s}^{-1}$. 
Table 3 - Mesh quality measures from OpenFOAM checkMesh utility

\begin{tabular}{|l|l|l|l|}
\hline & $\begin{array}{l}\text { Maximun } \\
\text { Aspect ratio }\end{array}$ & $\begin{array}{l}\text { Average } \\
\text { Mesh non-orthogonality }\end{array}$ & $\begin{array}{l}\text { Maximum } \\
\text { Skewness }\end{array}$ \\
\hline Before deformation & 6 & 6.4 & 0.98 \\
\hline Maximal deformation & 8 & 6.8 & 0.98 \\
\hline
\end{tabular}




\section{LIST OF FIGURE CAPTIONS}

Figure 1 - Free surface profiles in the tank with and without the plate.

Figure 2 - Velocity vectors, normalized vorticity field and free surface profiles from OpenFOAM simulations with or without the plate. Axes are distance in meters.

Figure 3 - Horizontal and vertical velocity one node ahead of the plate $(K C=6.3)$ at a submergence of $0.25 \mathrm{~m}$; a sinusoidal fit of the horizontal velocity is also shown.

Figure 4 - Estimation of starting and ending times of horizontal force cycles: in (a), minima of $\int \eta^{2} d x$ are shown with star symbols, and in (b), Points A and B denote the start and end of a force cycle normalized here as in Equation (2).

Figure 5-2-D grid convergence results at $K C=6.3$.

Figure 6 - Comparison of drag and inertia coefficients $\left(C_{d}\right.$ and $\left.C_{m}\right)$ for 2-D plates: measurements (Keulegan and Carpenter, 1958), and estimates using OpenFOAM numerical wave tank.

Figure 7 - Experimental results for the drag coefficients $\left(C_{d}\right)$ of oscillating circular disks at low $\beta$ (Figure 4 in Minamizawa and Endoh, 1984); numbers in the legend refer to $K C$; also shown here are estimates for all thin circular disk simulations using OpenFOAM.

Figure 8 - Detail of radial cross section for the selected 3-D mesh; numerical cell vertices are red lozenges and the blue area is occupied in this case by a portion of the disk.

Figure 9 - 3-D grid convergence results for the oscillations of a submerged circular disk $(K C=1.2$ and frequency of $1 \mathrm{~Hz})$. 
Figure 10 - (a) Detail of radial cross section for the higher-resolution 3-D mesh in thick disk $(\zeta=1 / 25)$ simulations; numerical cell vertices are red dots and the blue area is occupied in this case by a portion of the disk; (b) overall view of mesh over the disk.

Figure 11 - Radiating wave field around an oscillating disk close to free surface.

Figure 12 - Selected experimental data and OpenFOAM numerical simulations for the reduced drag coefficient $\left(C_{b}\right)$ of thin oscillating circular disks as a function of $K C$; numbers in the legend refer to $\beta$.

Figure 13 - Selected experimental data and OpenFOAM numerical simulations for the reduced added-mass coefficient $\left(A^{\prime}\right)$ of thin oscillating circular disks as a function of $K C$; numbers in the legend refer to $\beta$.

Figure 14 - Selected experimental data and OpenFOAM numerical simulations for the reduced drag coefficient $\left(C_{b}\right)$ of oscillating circular disks of various thickness ratios as a function of $K C$; numbers in the legend refer to $\zeta$.

Figure 15 - Velocity vectors and normalized vorticity field from OpenFOAM simulations with $\zeta=1 / 25$ and $K C=0.05$; subfigures (a) to (h) correspond to 8 equidistant times through the oscillation cycle, with the disk at its topmost position in (a); vector scale (red arrow) is $3.5 \mathrm{~cm} / \mathrm{s}$.

Figure 16 - Same as Figure 15 with $\zeta=1 / 50$ and $K C=0.2$ (note that the thickness ratio varies by changing the disk diameter); vector scale (red arrow) is $7.9 \mathrm{~cm} / \mathrm{s}$.

Figure 17 - Selected experimental data and OpenFOAM numerical simulations for the reduced drag coefficient $\left(C_{b}\right)$ of thin oscillating square plates as a function of $K C$; numbers in the legend refer to $\beta$. 


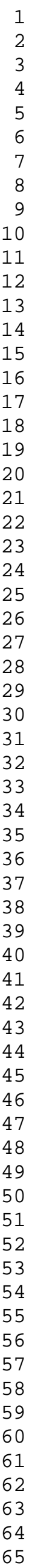

figures

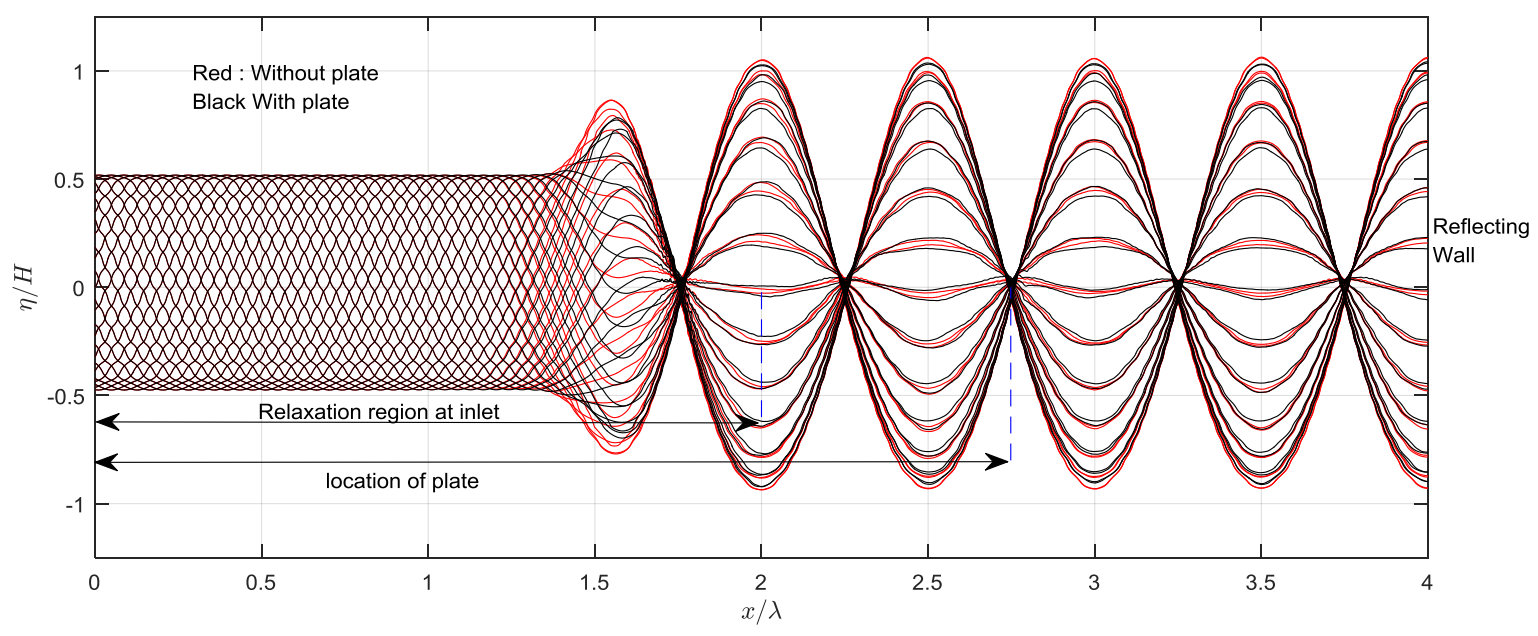

fig 1 
fig 2

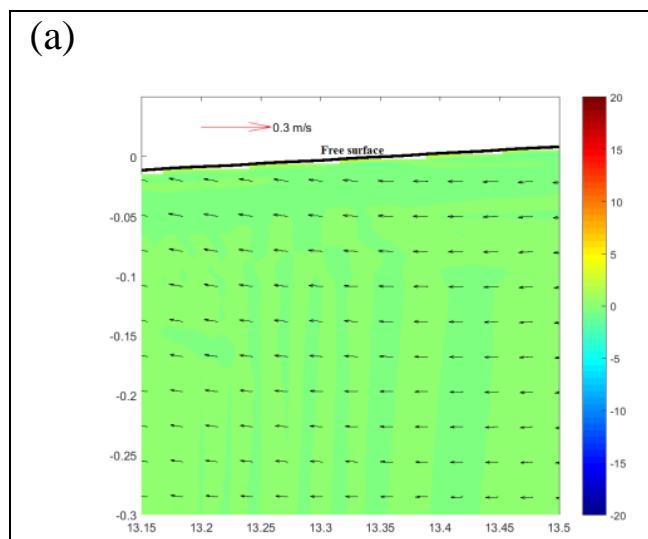

(b)

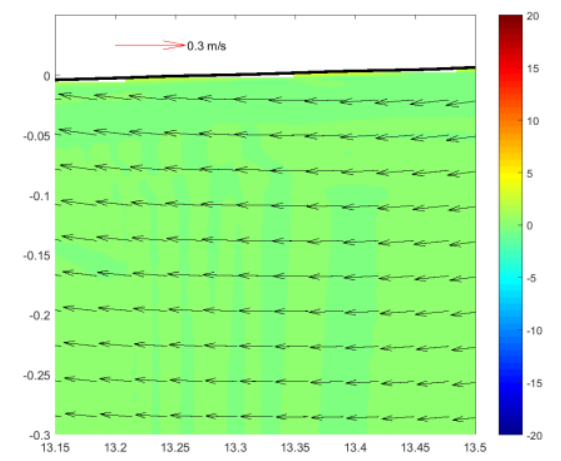

(c)

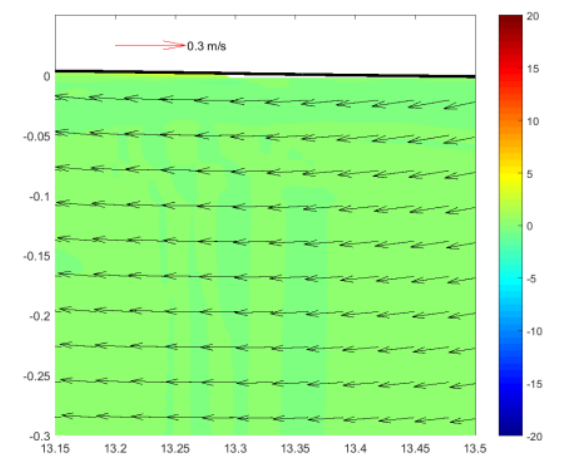

(d)

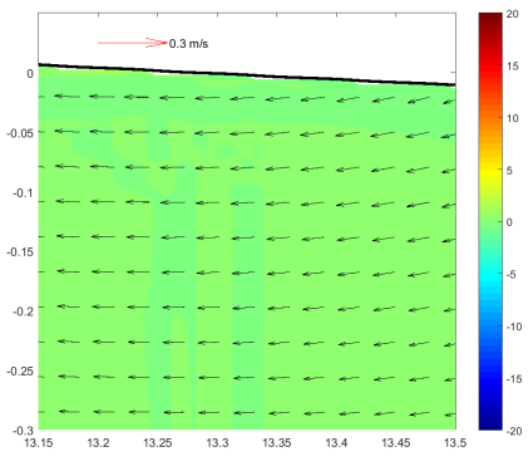

(i)

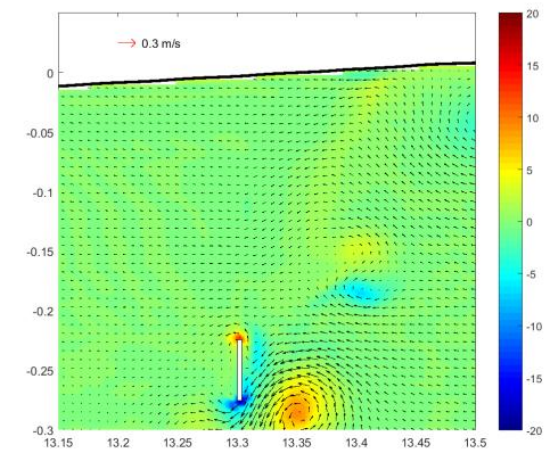

(j)

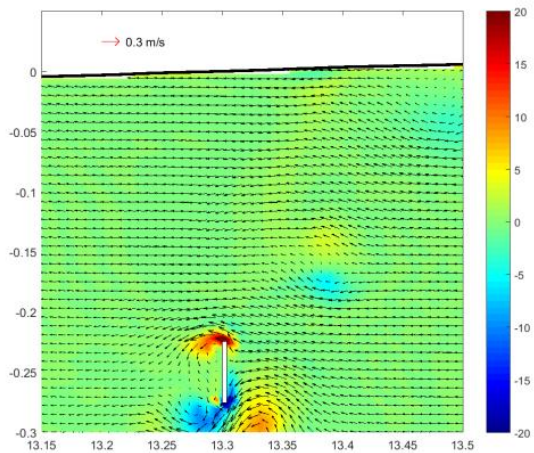

(k)

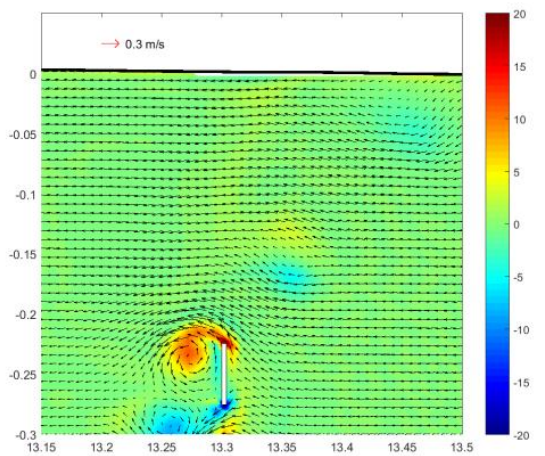

(1)

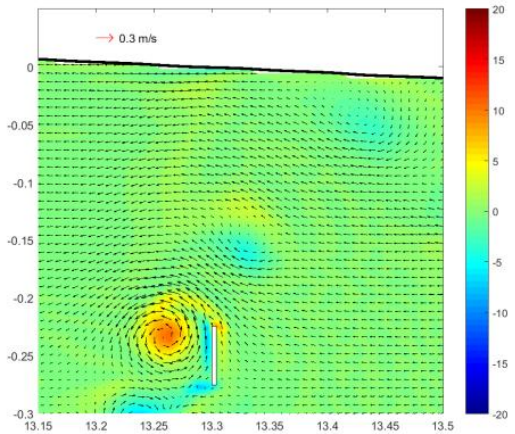




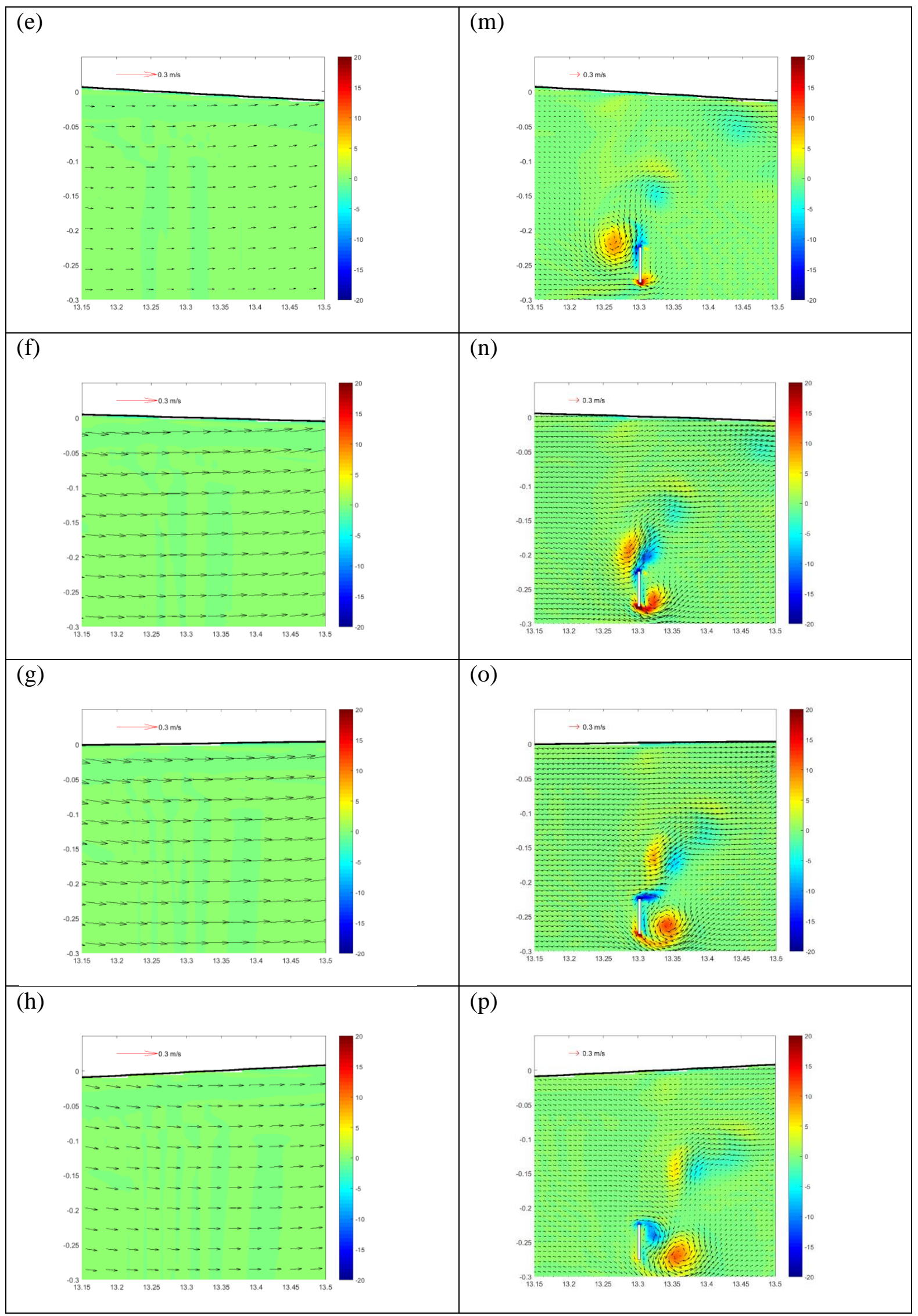


fig 3

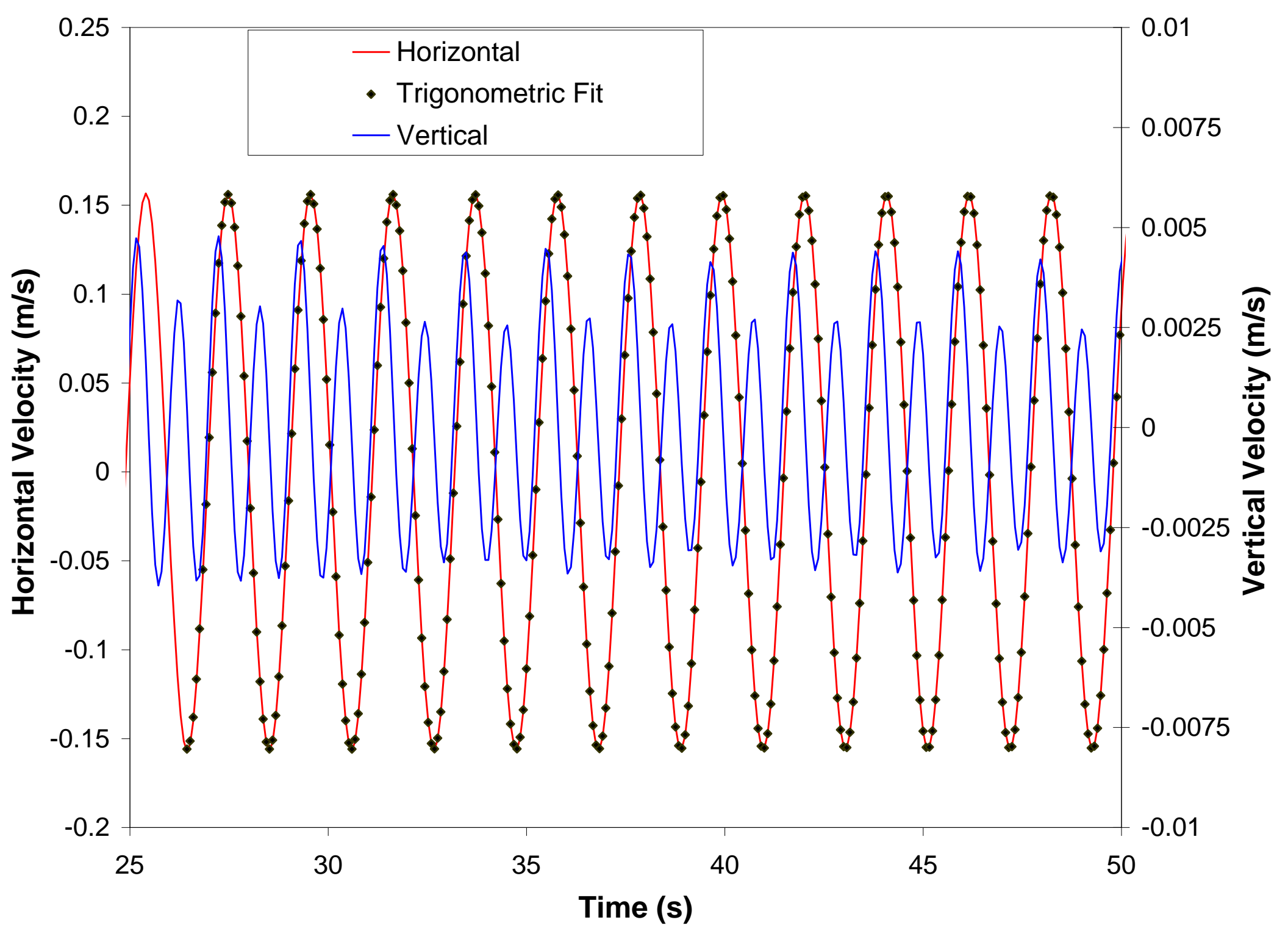


fig 4
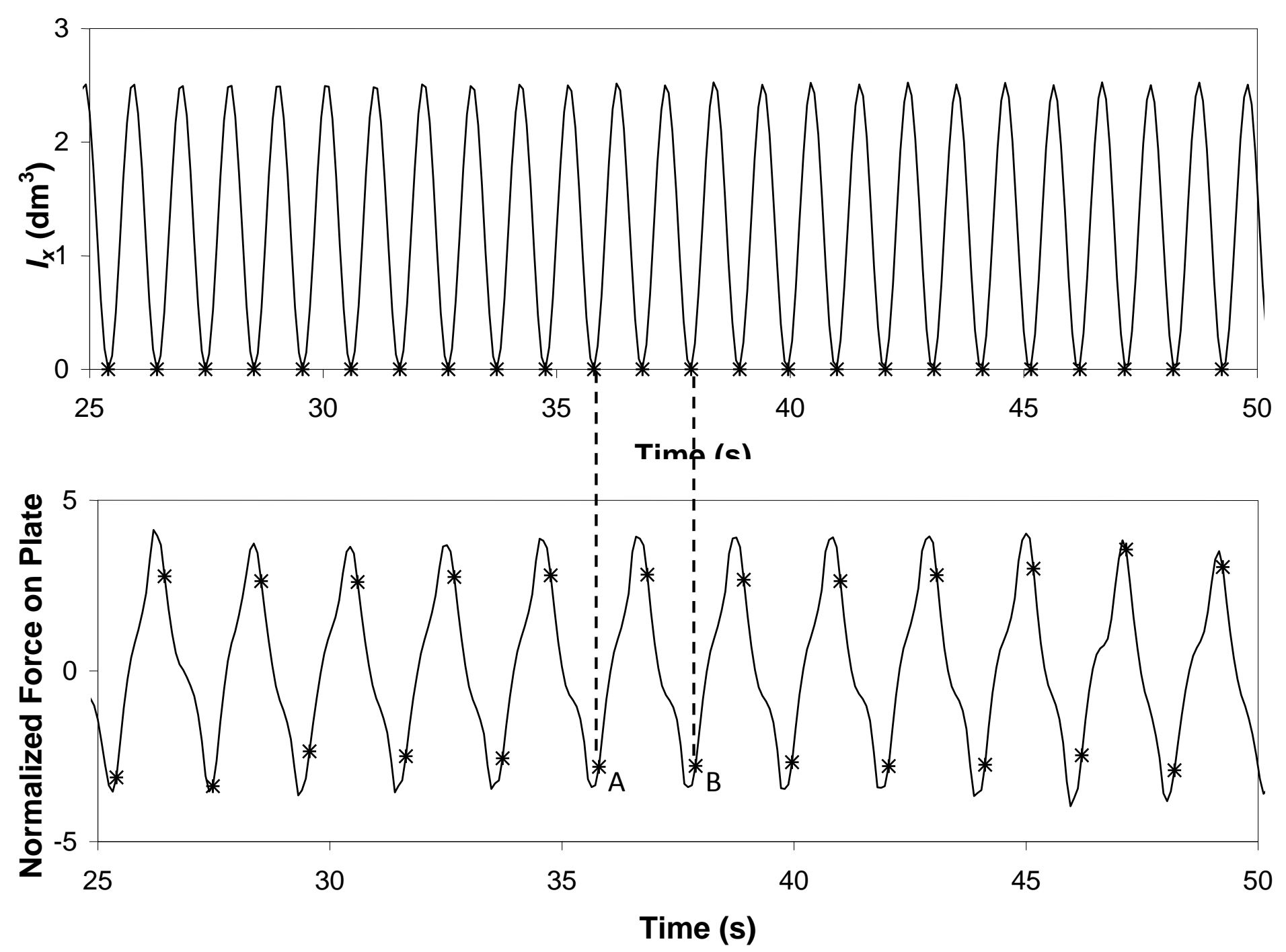
fig 5

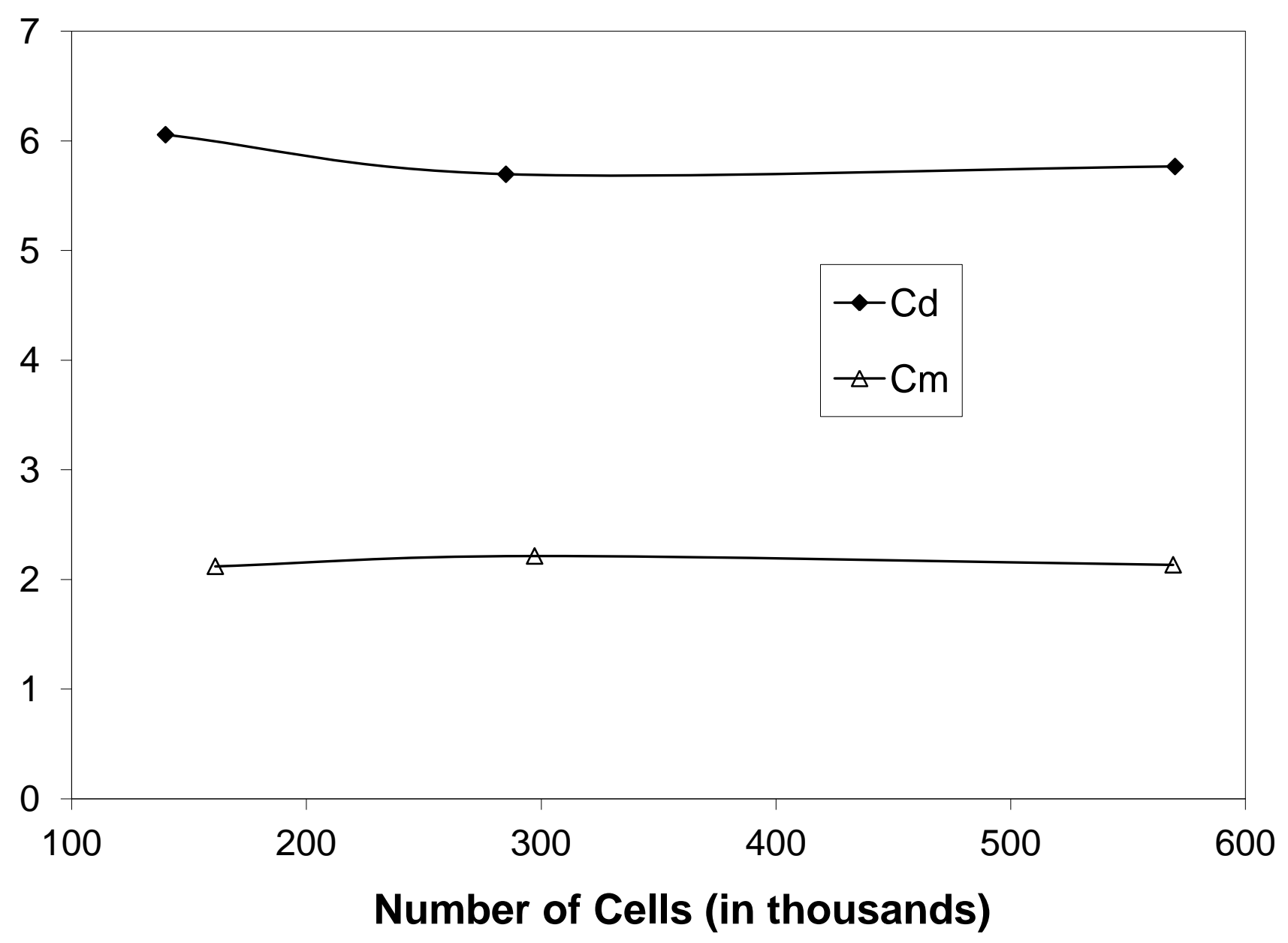


fig 6

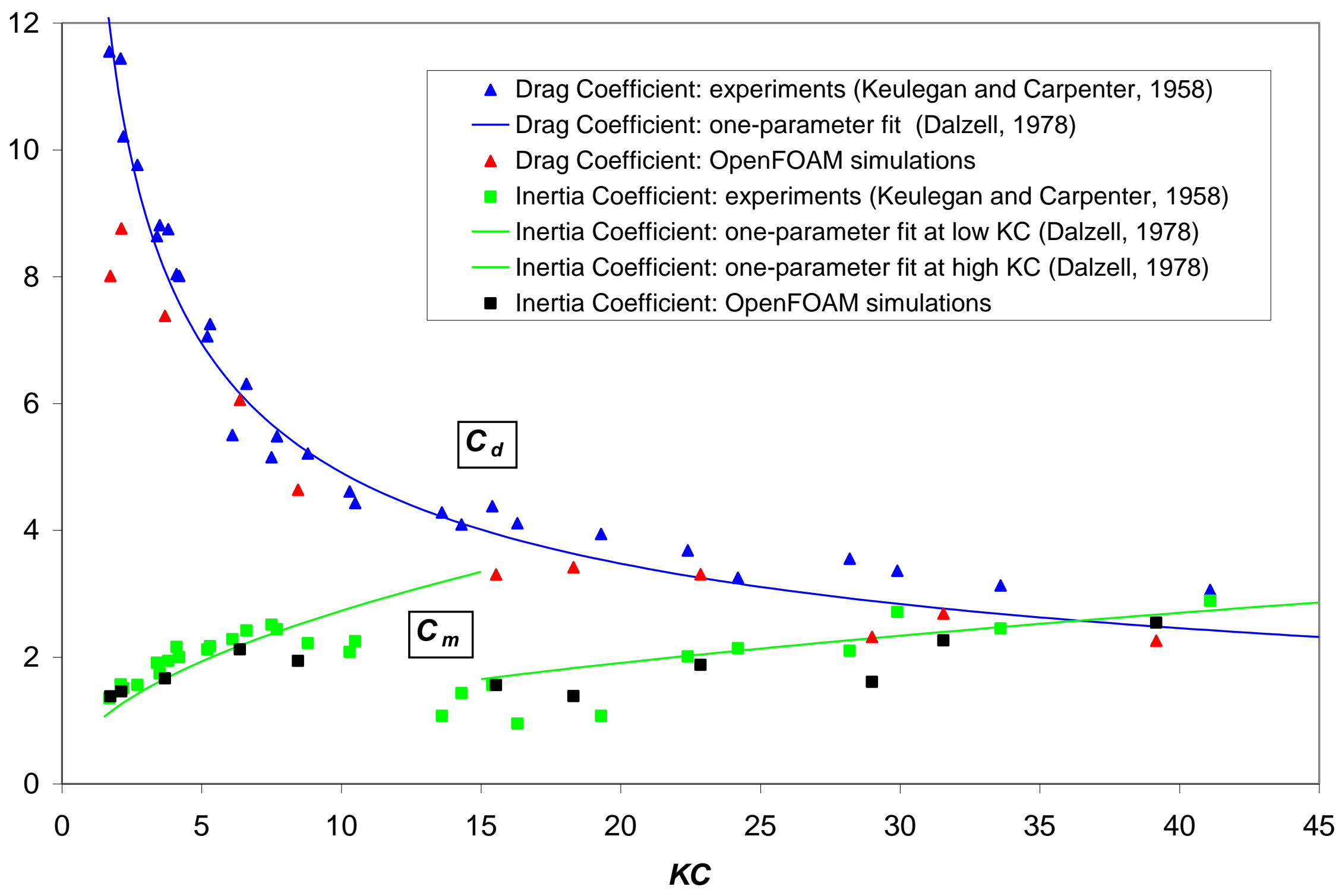


fig 7

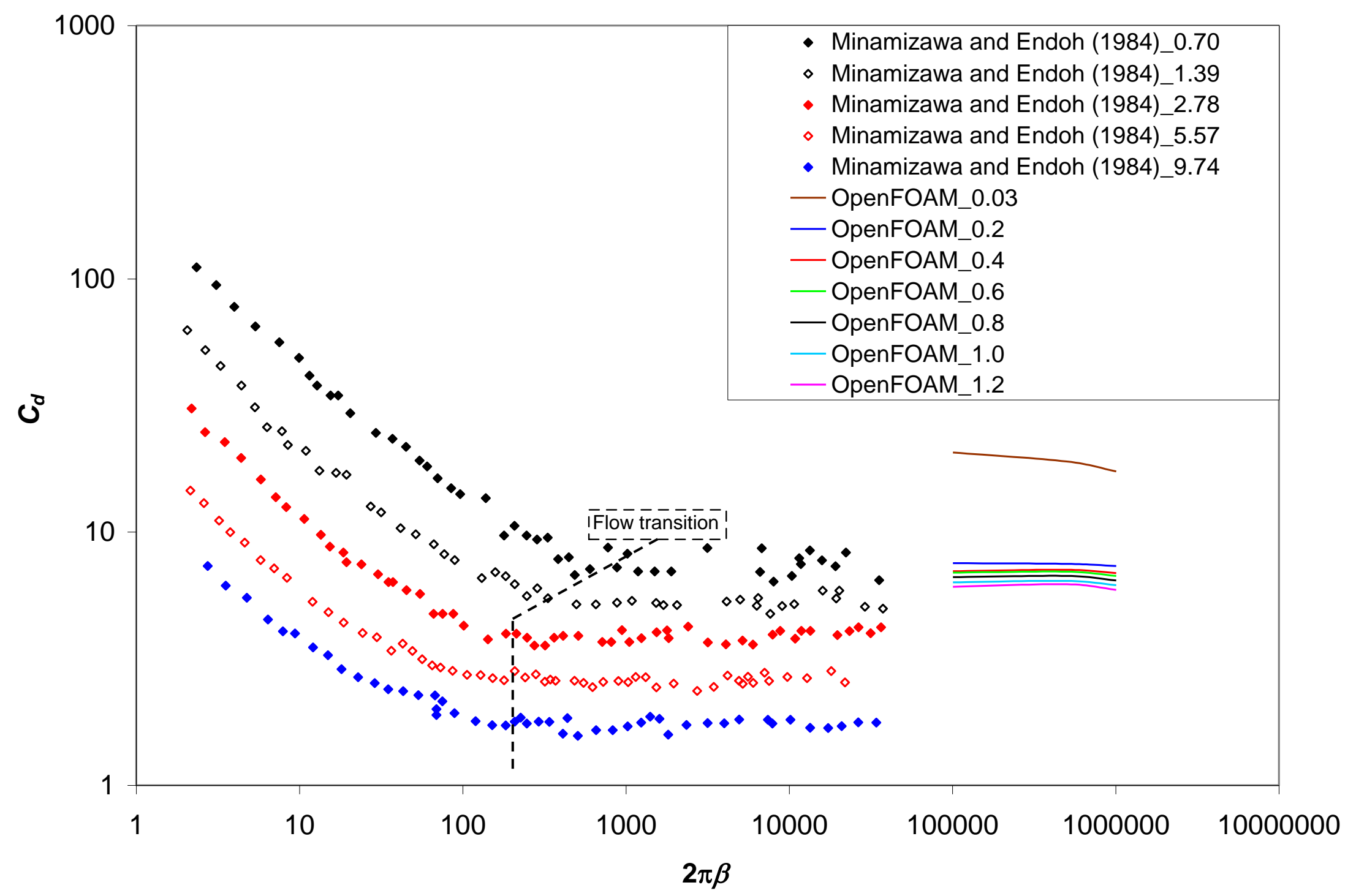


fig 8

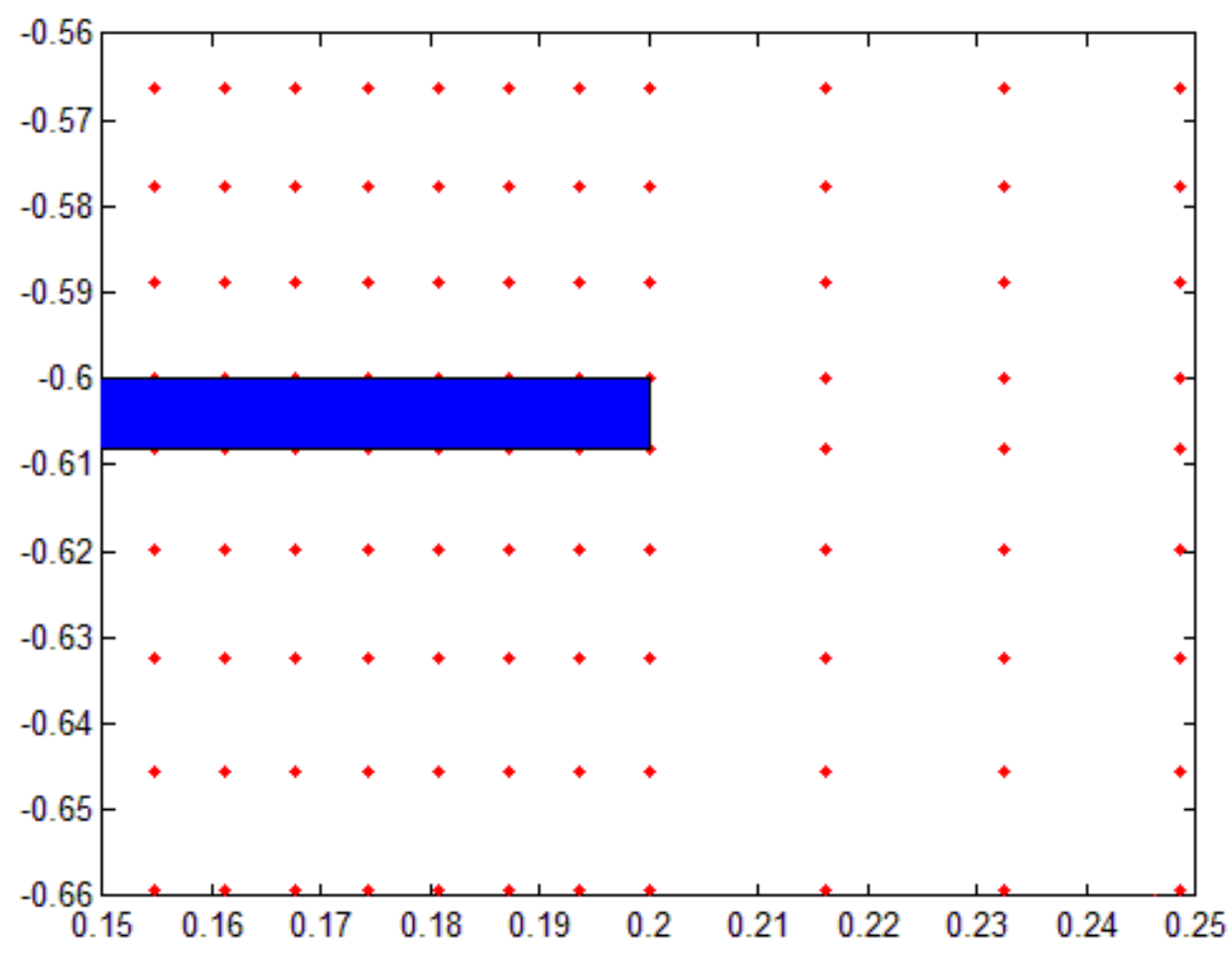

fig 9

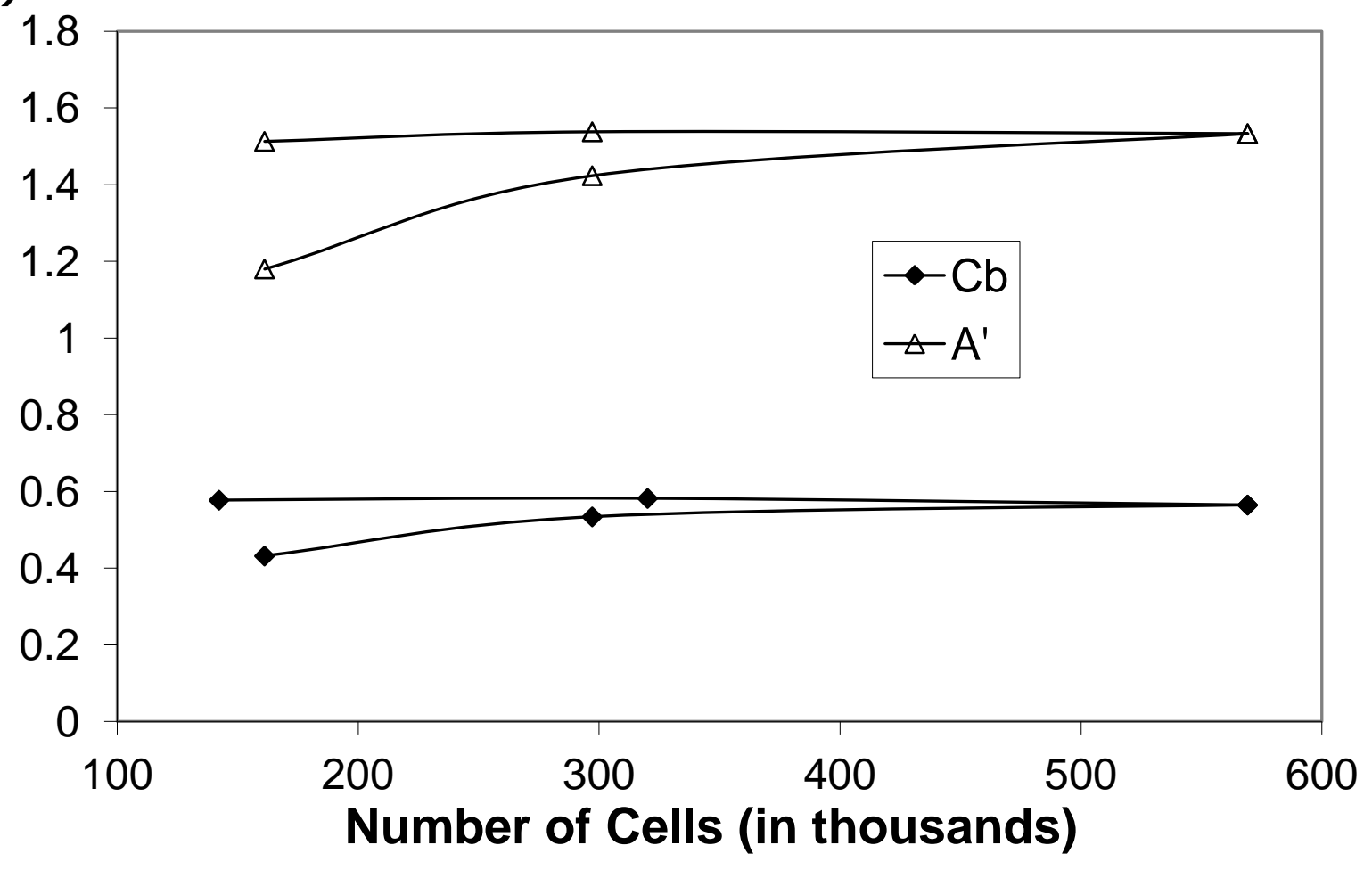


fig 10
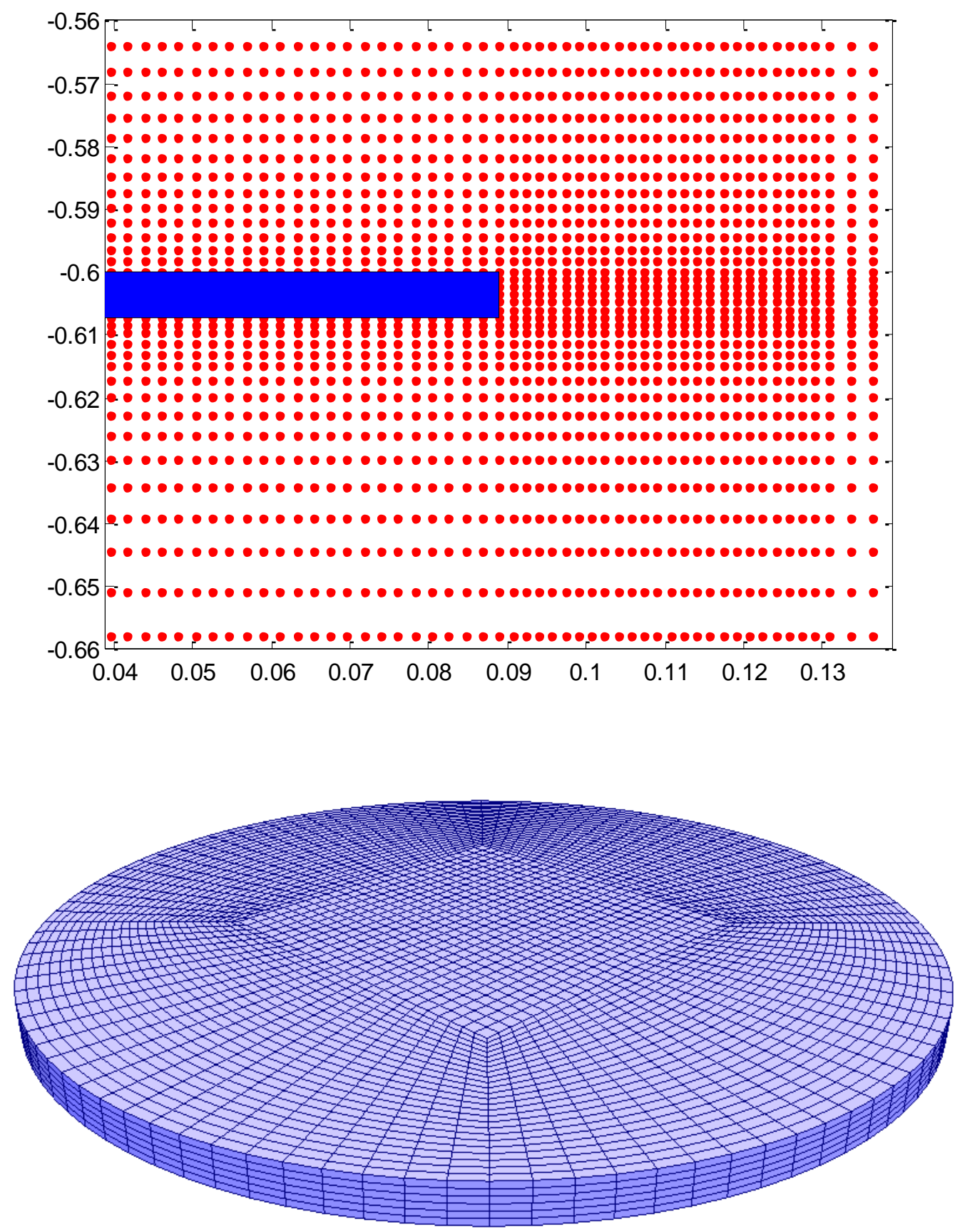
fig 11

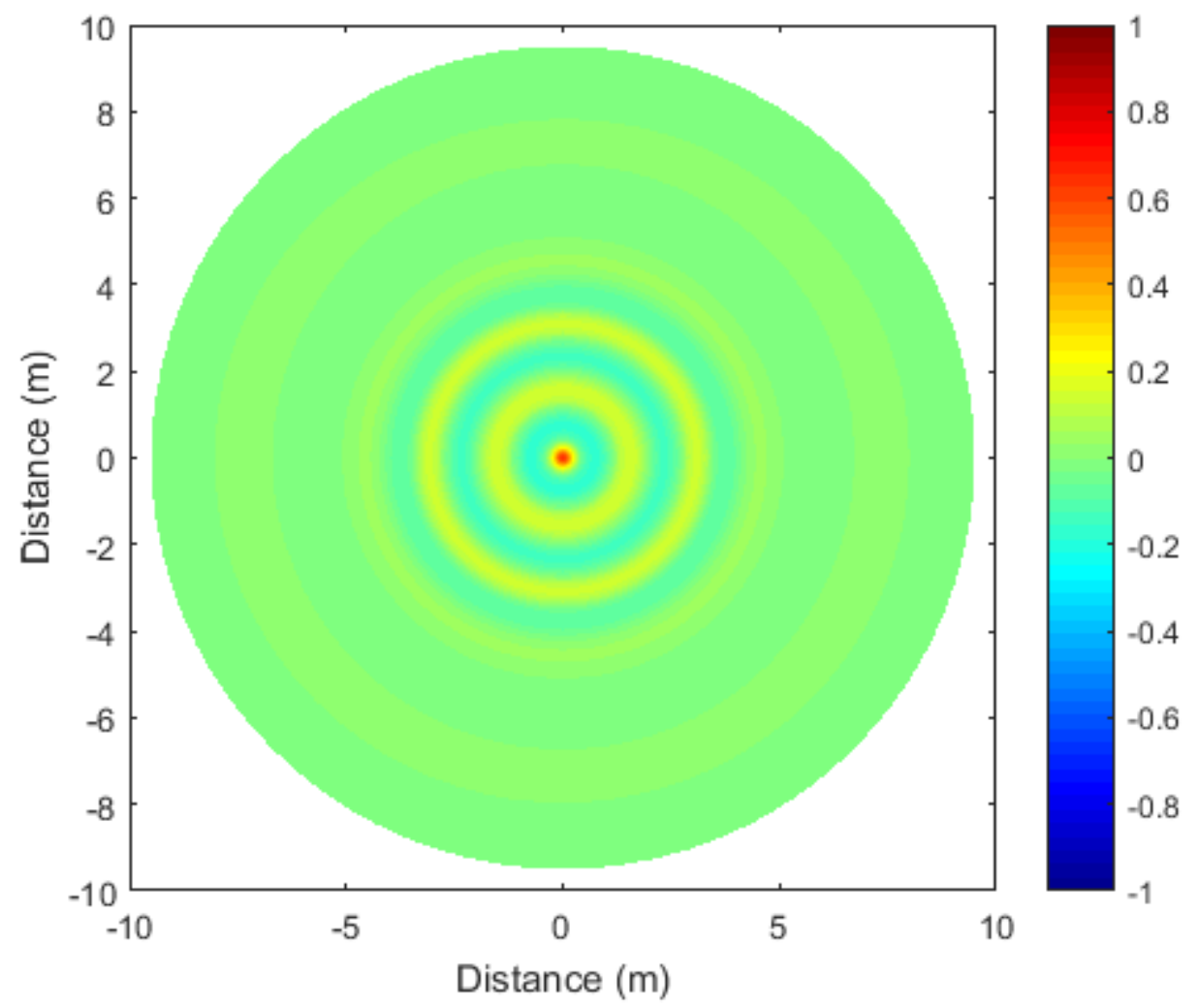


fig 12

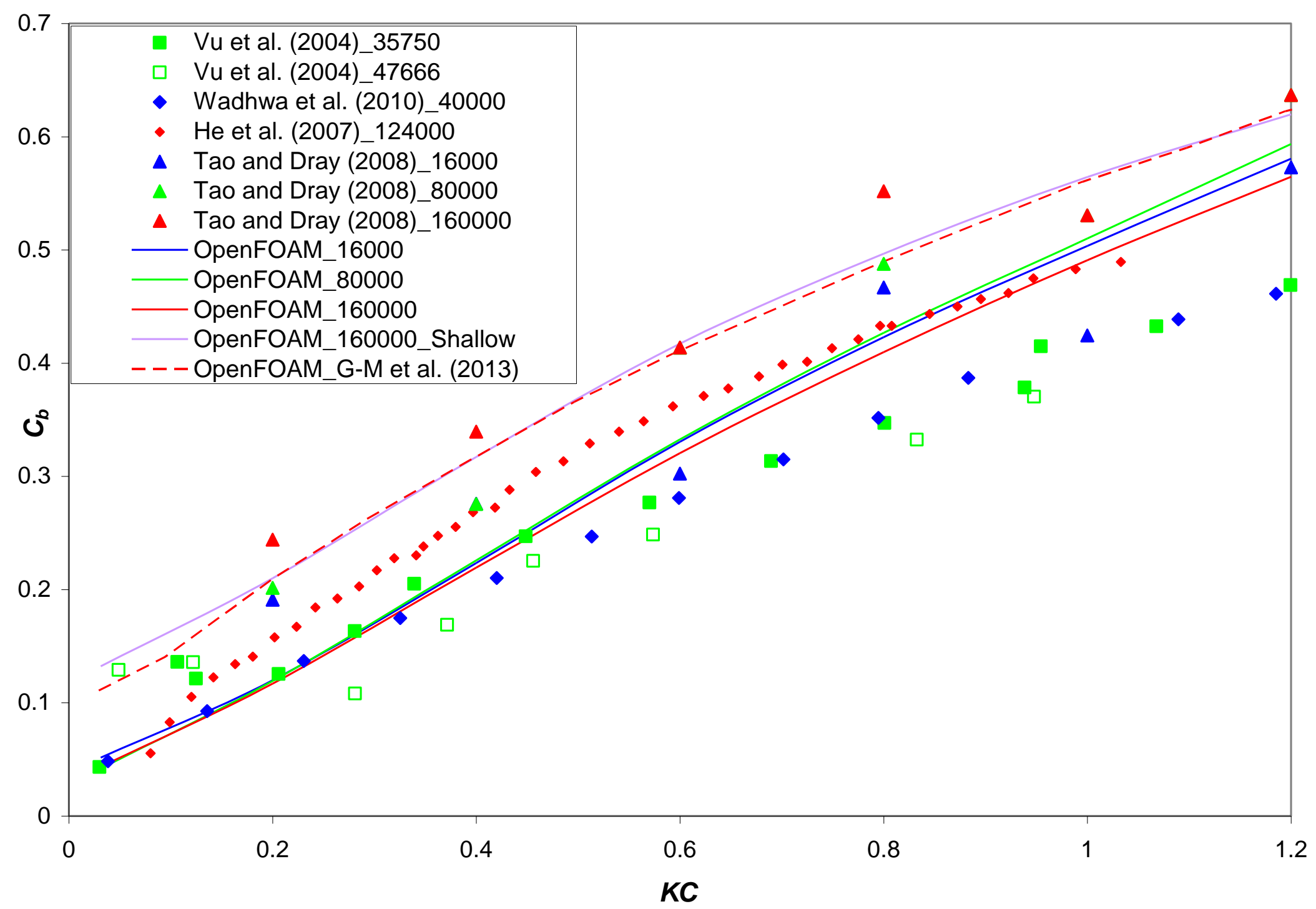


fig 13

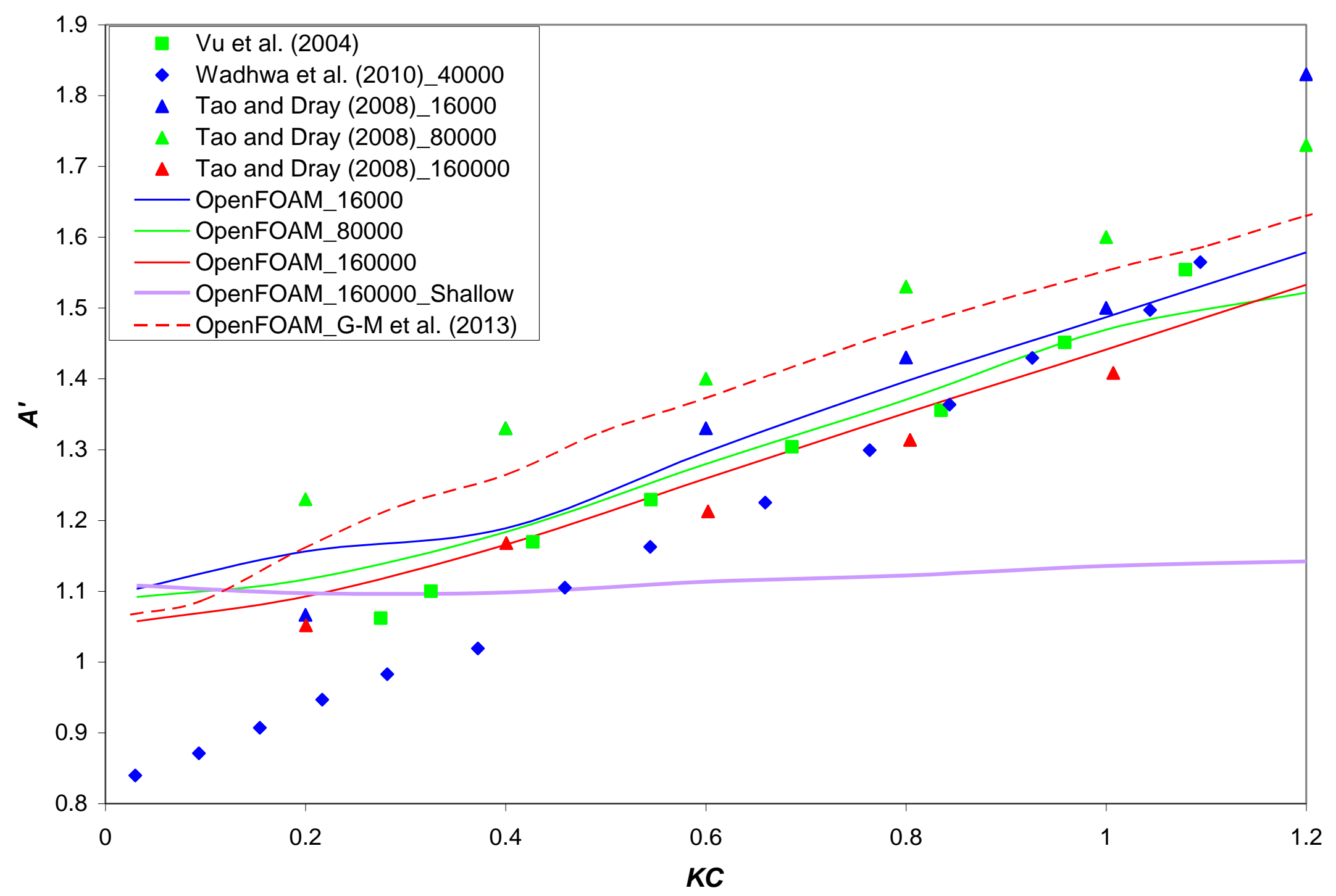


fig 14

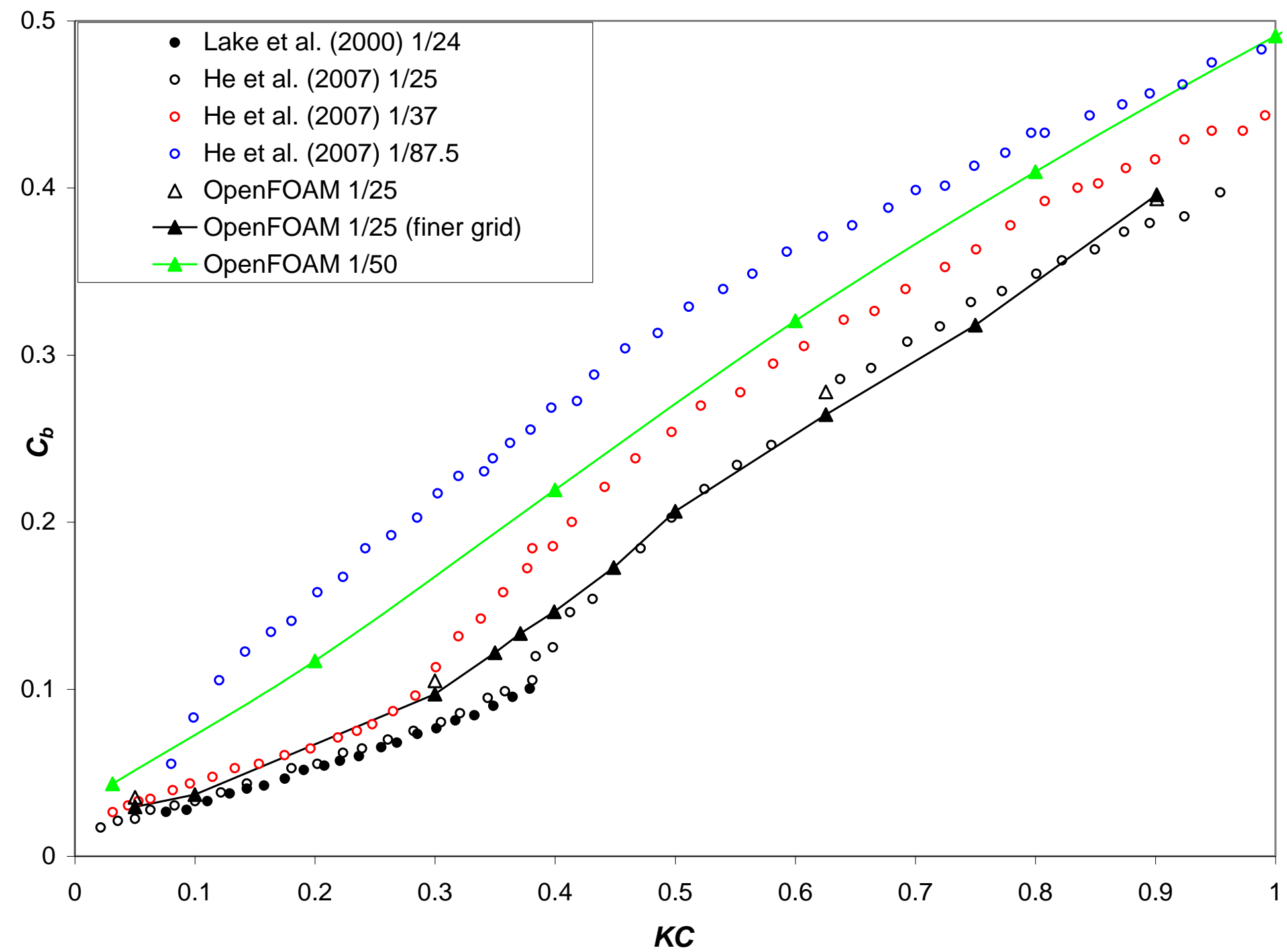



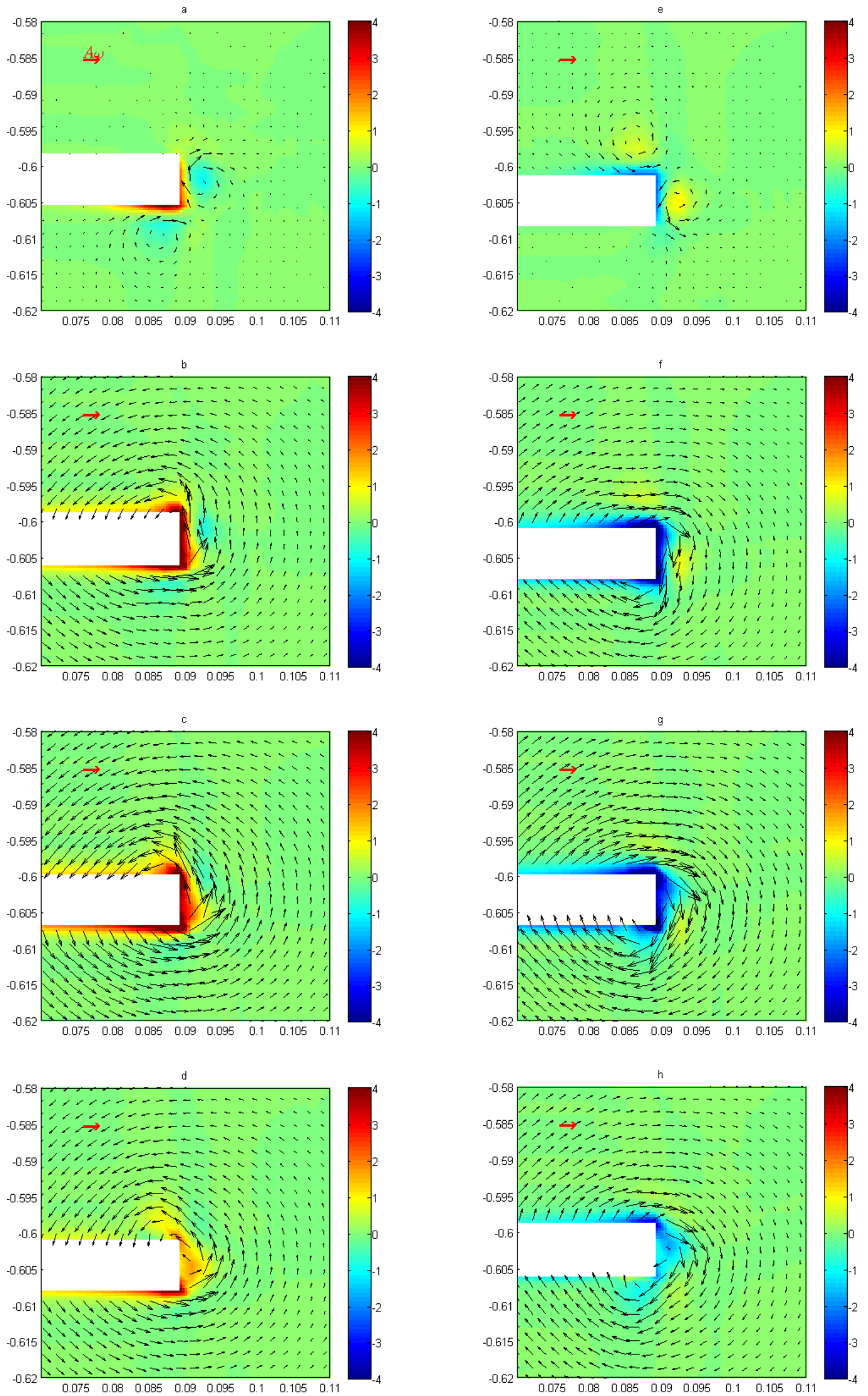
fig 16
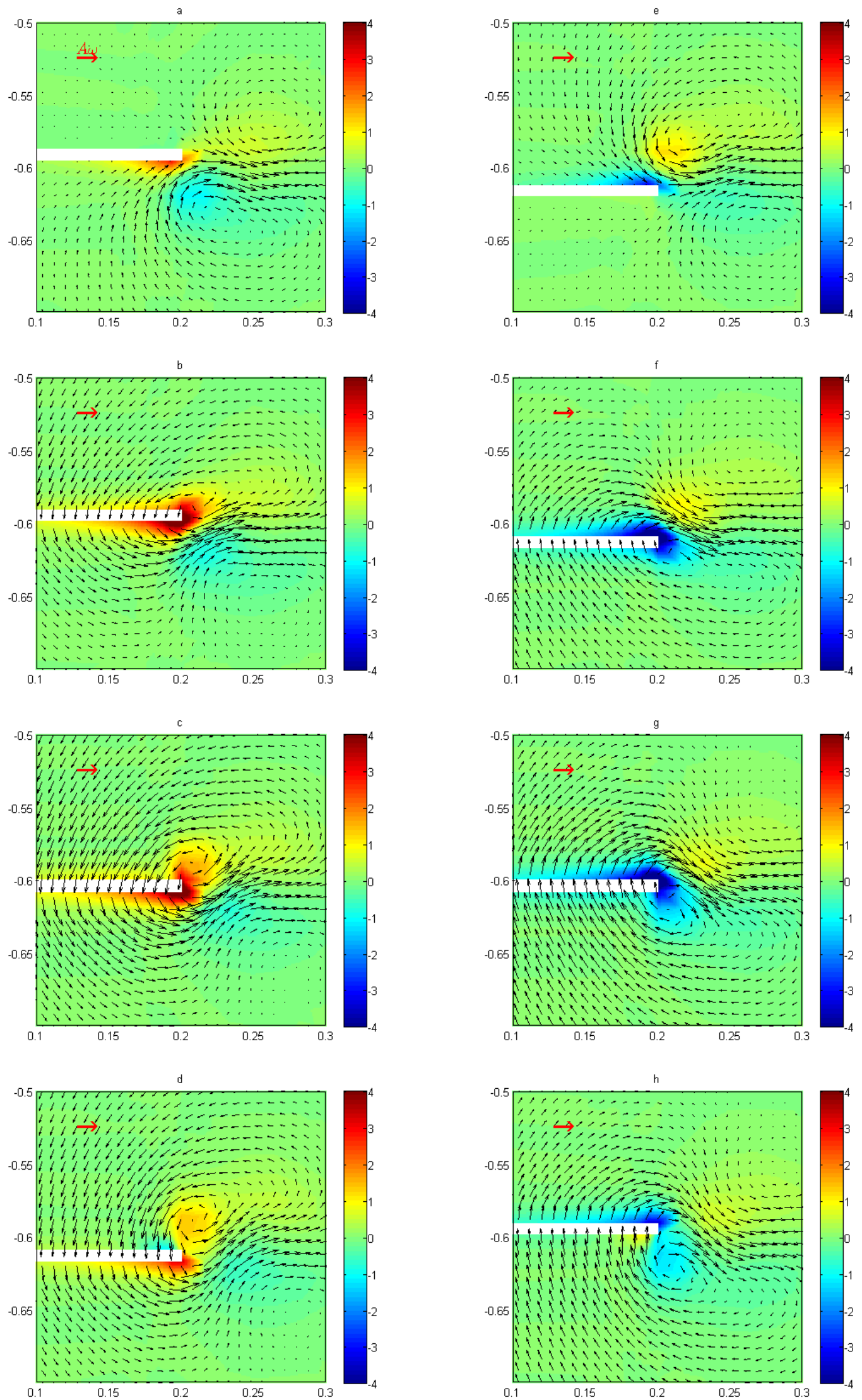
fig 17

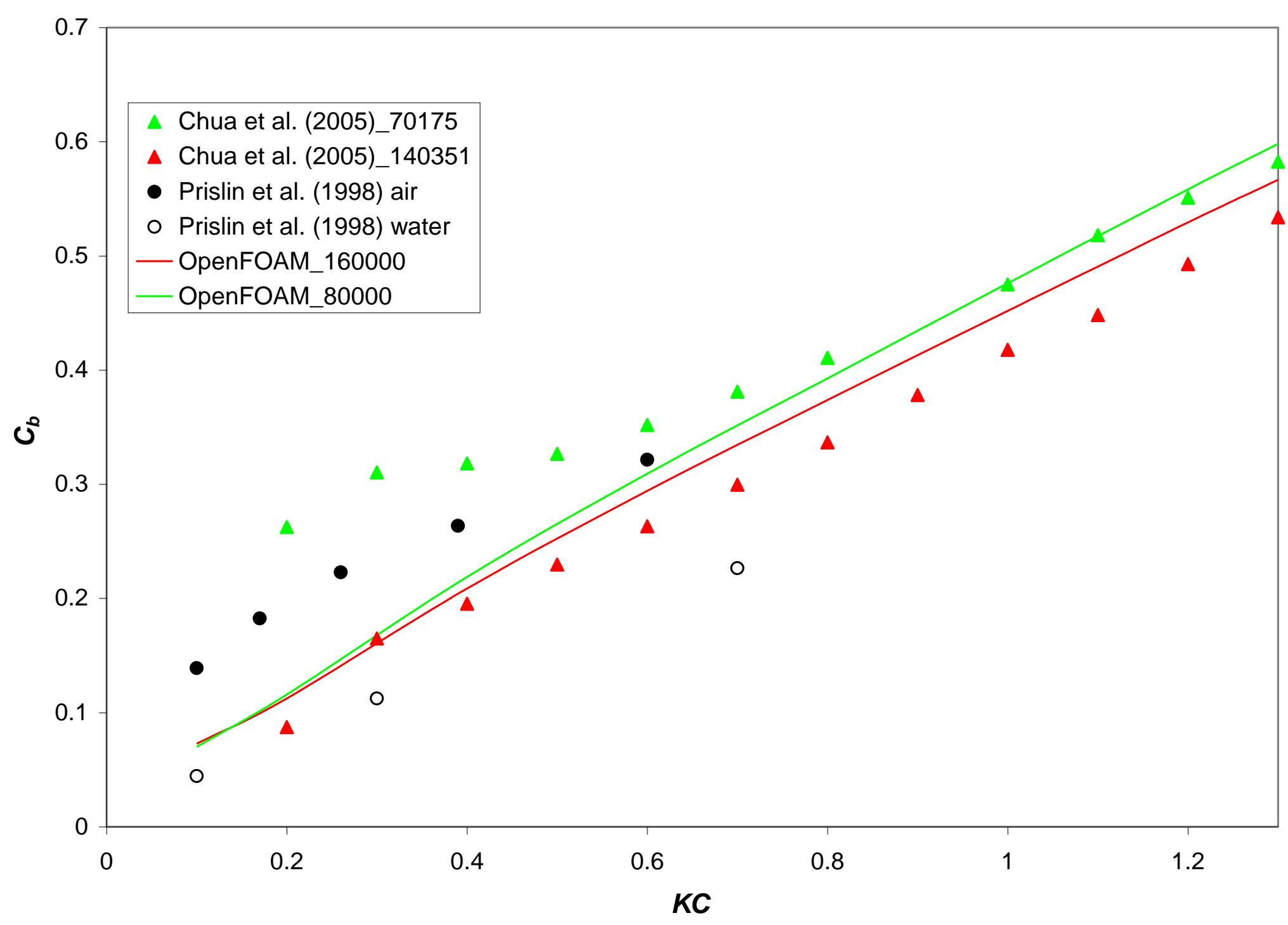

